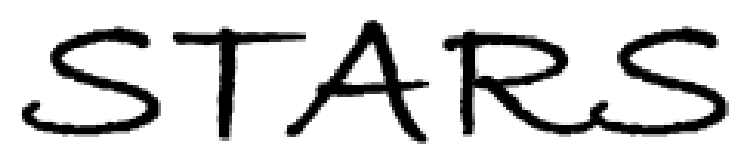

University of Central Florida

STARS

\title{
Structured Light-Field Focusing 3D Density Measurements of A Supersonic Cone
}

Ryonosuke Ozawa

University of Central Florida

Part of the Aerodynamics and Fluid Mechanics Commons

Find similar works at: https://stars.library.ucf.edu/etd

University of Central Florida Libraries http://library.ucf.edu

This Masters Thesis (Open Access) is brought to you for free and open access by STARS. It has been accepted for inclusion in Electronic Theses and Dissertations by an authorized administrator of STARS. For more information, please contact STARS@ucf.edu.

\section{STARS Citation}

Ozawa, Ryonosuke, "Structured Light-Field Focusing 3D Density Measurements of A Supersonic Cone" (2018). Electronic Theses and Dissertations. 5823.

https://stars.library.ucf.edu/etd/5823

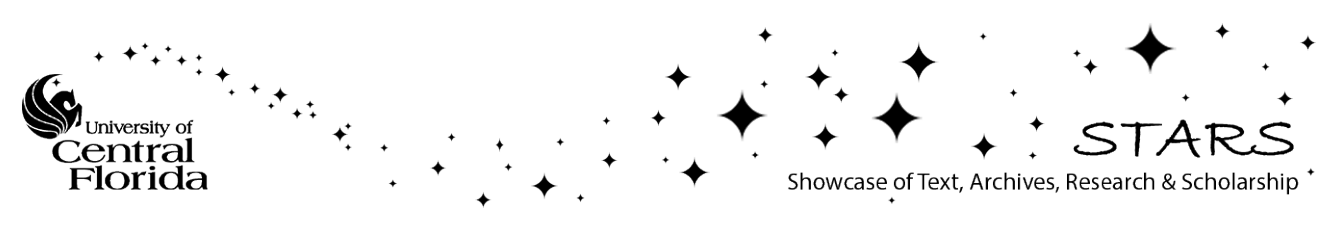




\title{
STRUCTURED LIGHT-FIELD FOCUSING 3D DENSITY MEASUREMENTS OF A SUPERSONIC CONE
}

\author{
by \\ RYONOSUKE WILLIAM OZAWA \\ B. S. Yokohama National University, 2015
}

A thesis submitted in partial fulfillment of the requirements for the degree of Master of Science in the Department of Mechanical and Aerospace Engineering in the College of Engineering and Computer Science at the University of Central Florida Orlando, Florida

Spring Term

2018

Major Professor: Kareem A. Ahmed 
C2018 Ryonosuke William Ozawa 


\begin{abstract}
This study describes three-dimensional (3D) quantitative visualization of density field in a supersonic flow around a cone spike. A measurement of the density gradient is conducted within a supersonic wind tunnel facility at the Propulsion and Energy Research Laboratory at the University of Central Florida utilizing Structured Light-Field Focusing Schlieren (SLLF). In conventional schlieren and Shadowgraph techniques, it is widely known that a complicated optical system is needed and yet visualizable area depends on an effective diameter of lenses and mirrors. Unlike these techniques, SLLF is yet one of the same family as schlieren photography, it is capable of non-intrusive turbulent flow measurement with relatively low cost and easy-to-setup instruments. In this technique, cross-sectional area in the flow field that is parallel to flows can be observed while other schlieren methods measure density gradients in line-of-sight, meaning that it measures integrated density distribution caused by discontinuous flow parameters. To reconstruct a 3D model of shock structure, two-dimensional (2D) images are pictured to process in MATLAB. The ultimate goal of this study is to introduce a novel technique of SLLF and quantitative 3D shock structures generated around a cone spike to reveal the interaction between free-stream flow and the high-pressure region.
\end{abstract}




\section{ACKNOWLEDGMENTS}

The author would like to acknowledge foremost my family. Without their continuous support and help during the pursuit of this endeavor, this work would not be possible. Miki Ozawa and Toshiko Ozawa, who raised me and contributed in enriching my career, I will be in debt for my entire life. Yuunosuke Ozawa, who shaped or formed my character, your advice and action taught me to keep my ambition. If not for Annalee Liao, who inspired and motivated me to keep pushing the limits of my ability to excel in life, this couldn't be accomplished. To my lab coworkers who dedicated their support through this, I cannot thank you enough. Last, the author also wishes to acknowledge my facilitator who guided me in this project, Dr. Kareem Ahmed. Your

extensive knowledge of the subject has stimulated me to pursue my career in aerospace engineering. Thank you all. 


\section{TABLE OF CONTENTS}

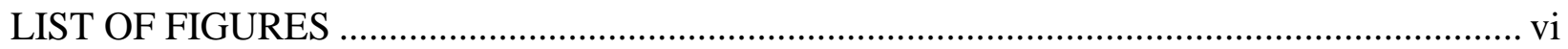

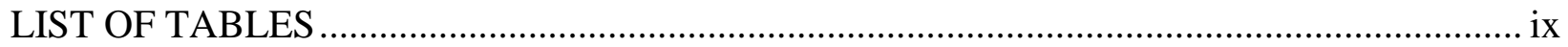

LIST OF ABBREVIATION (OR) ACRONYMS ……………...........................................

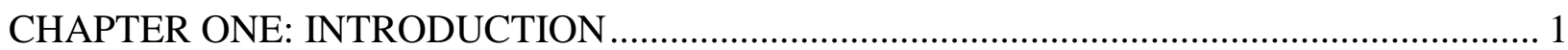

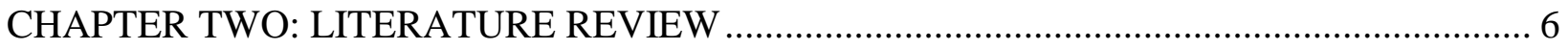

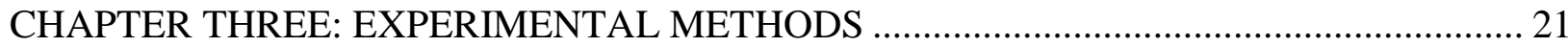

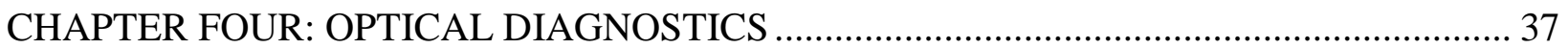

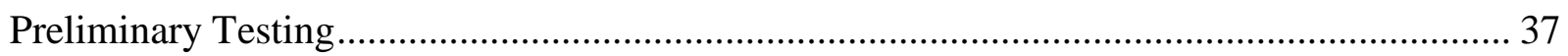

Supersonic Wind Tunnel Testing...................................................................................... 43

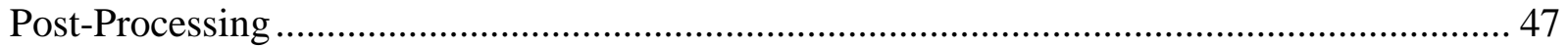

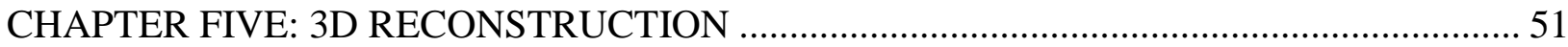

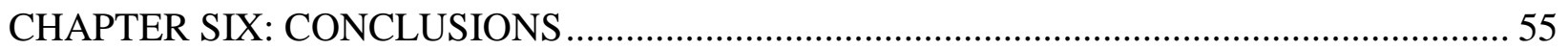

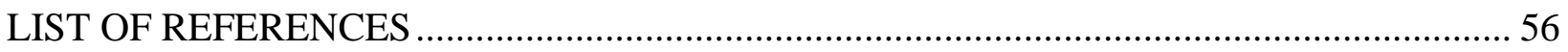




\section{LIST OF FIGURES}

Figure 1: Two-dimensional Ramp Intakes at Different Mach Number ...................................... 2

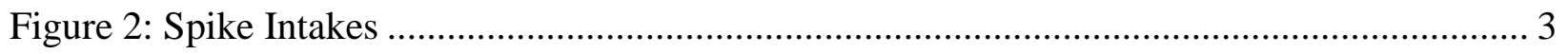

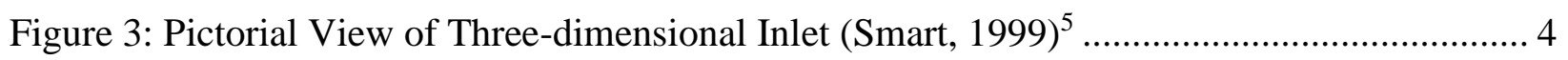

Figure 4: Conventional Schlieren Schematic ............................................................... 7

Figure 5: Z-type Schlieren Schematic........................................................................ 7

Figure 6: Sharp Focusing Schlieren Optical System ..................................................... 9

Figure 7: Focused-Schlieren Images of $\mathrm{CO}_{2}$ Jet at Four Axial Locations (Positive X Directions)

Figure 8: Focused-Schlieren Images of $\mathrm{CO}_{2}$ Jet at Four Axial Locations (Negative X Directions)

Figure 9: Shock Wave Profiles at Different Focal Location................................................. 12

Figure 10: Shock Wave Profile with the Conventional Schlieren Method............................... 13

Figure 11: Shock Wave Profile with the Conventional and the Focusing Schlieren Method ...... 13

Figure 12: Flow Visualization around the Double Wedge Airfoil Model ................................. 14

Figure 13: Shock Wave Profile on the Double Wedge Airfoil Model $\left(\alpha=0^{\circ}\right) \ldots \ldots \ldots \ldots \ldots \ldots \ldots \ldots . . . . . . . . . .15$

Figure 14: Schematic View of the Direct-Connect Dual-Mode Scramjet with a Ramp Injector . 16

Figure 15: Typical Focusing-Schlieren Images under Cold Flow Condition: a fuel off and b fuel

on.

Figure 16: Typical Focusing Schlieren Images for the Case with Igniter On: a before flow path

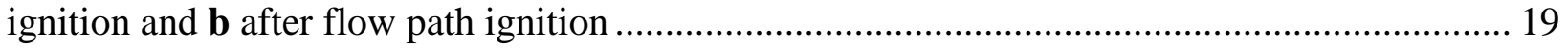

Figure 17: Typical Focusing-Schlieren Image for the Case of Fuel-Air Reacting ..................... 19 


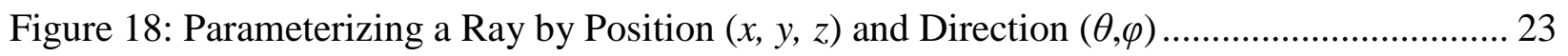

Figure 19: Conceptual Schematic of a Plenoptic Camera ................................................. 24

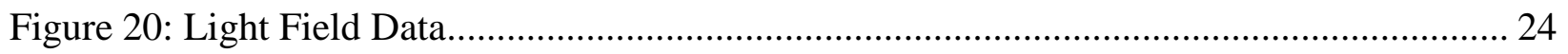

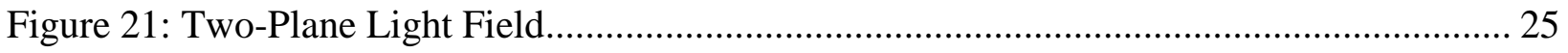

Figure 22: Two Sub-Aperture Photographs with Vertical Parallax........................................ 26

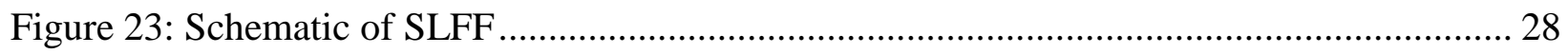

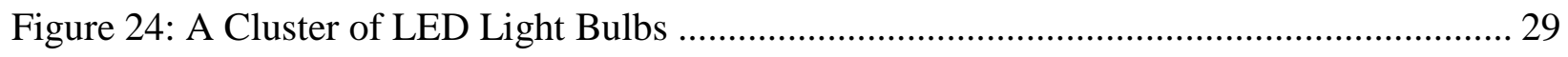

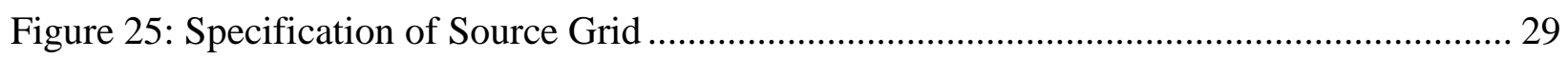

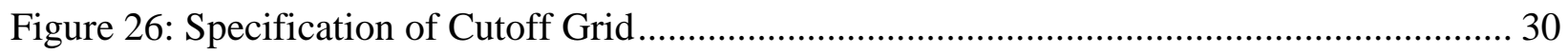

Figure 27: Actual Setup of Current System ................................................................. 31

Figure 28: Different Optical Configurations Tested ....................................................... 32

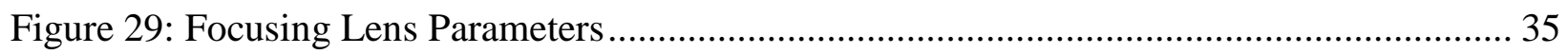

Figure 30: Light Source Offset Effect on the Effective F-number ....................................... 35

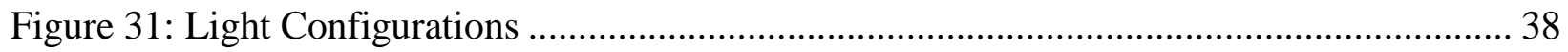

Figure 32: DOF for Outer Diameter Light Configuration .................................................... 41

Figure 33: DOF for Inner Diameter Light Configuration ................................................ 41

Figure 34: DOF for Center Light Configuration............................................................ 42

Figure 35: Under-Expanded Jet with 300mm(left) \& 250mm(right) Focal Length ................... 42

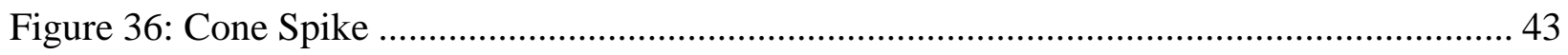

Figure 37: Sliced Shock lines at Each Translation ....................................................... 45

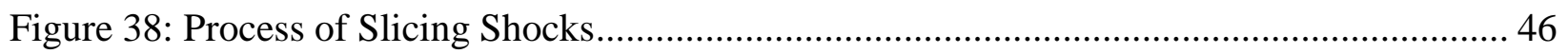

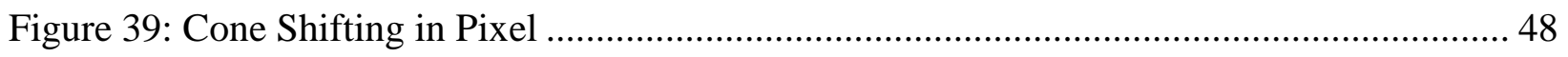




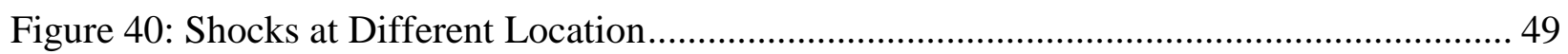

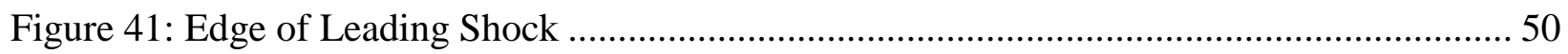

Figure 42: 3D Reconstruction of Shock Structure (XYZ view) ......................................... 52

Figure 43: 3D Reconstruction of Shock Structure (XY view \& XZ view) ............................. 52

Figure 44: 3D model with an Intersecting Plane .......................................................... 53

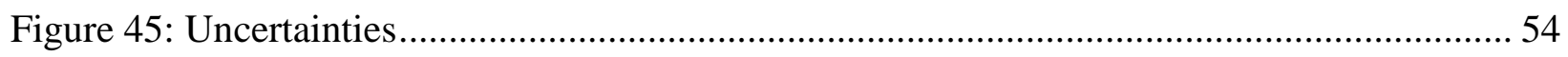




\section{LIST OF TABLES}

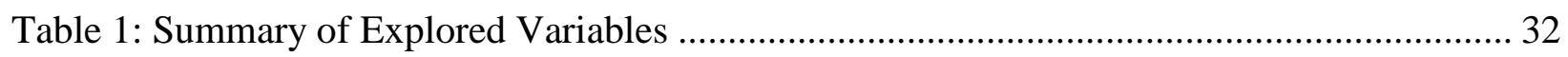

Table 2: DOF Measurements for Each Light Configuration ................................................ 39

Table 3: DOF Measurements for Each Light Configuration ................................................ 40 


\section{LIST OF ABBREVIATION (OR) ACRONYMS}

Background Oriented Schlieren $\quad$ BOS

Depth of Focus

DOF

Light Source Offset Distance

OS

Structured Light-Field Focusing Schlieren

SLFF 


\section{CHAPTER ONE: INTRODUCTION}

Most modern supersonic aircraft are powered by high-speed engines such as scramjet and ramjet engines. There are different types of engines; however, all of them have some part in common. The inlet, for instance, also known as intake, is one of the critical elements for the supersonic propulsion system to bring free-stream air into the engine. The performance of inlets is evaluated by the rate of total pressure recovery, operation stability and aerodynamic resistance from the viewpoint of compatibility and expected thrust as well. To maximize the performance, many types of inlets have been invented and designed. There are mainly two types of inlets for the specifically supersonic propulsion system. One is $2 \mathrm{D}$ ramp intake that is normally designed to have a rectangular entrance with an oblique shape. The forward edge is designed to generate an oblique shock and its position can be adjusted to hit the rearward edge on the other side. Either one or both of these edges can be shifted up and down to have the shock wave generated by the forward edge hit another. It is also capable of adjusting mass flow rate over a wide range by changing the area of cross-section. While this inlet is capable of operating at various speeds, it needs massive structural reinforcements to stay in shape. The pressure in the intake is considerably larger than the ambient at supersonic velocity, thus it is relatively heavy and complex. On the other hand, spike intakes which have a cone-shaped spike in the center have light weight and compactness due to its structural simplicity. Creating additional and steeper shock waves to decelerate the incoming flow over a sequence of shocks is also possible by varying cone angle for typical spike intakes. In a few of this intake, a translation mechanism that is responsible for shifting the spike back and forth to adjust the shock position and intake cross-section area is contained. While there are some 
significant advantages of this intake, it is widely known as drawbacks that it is likely to have longer internal duct and limited mass flow rate when operated at low speed.

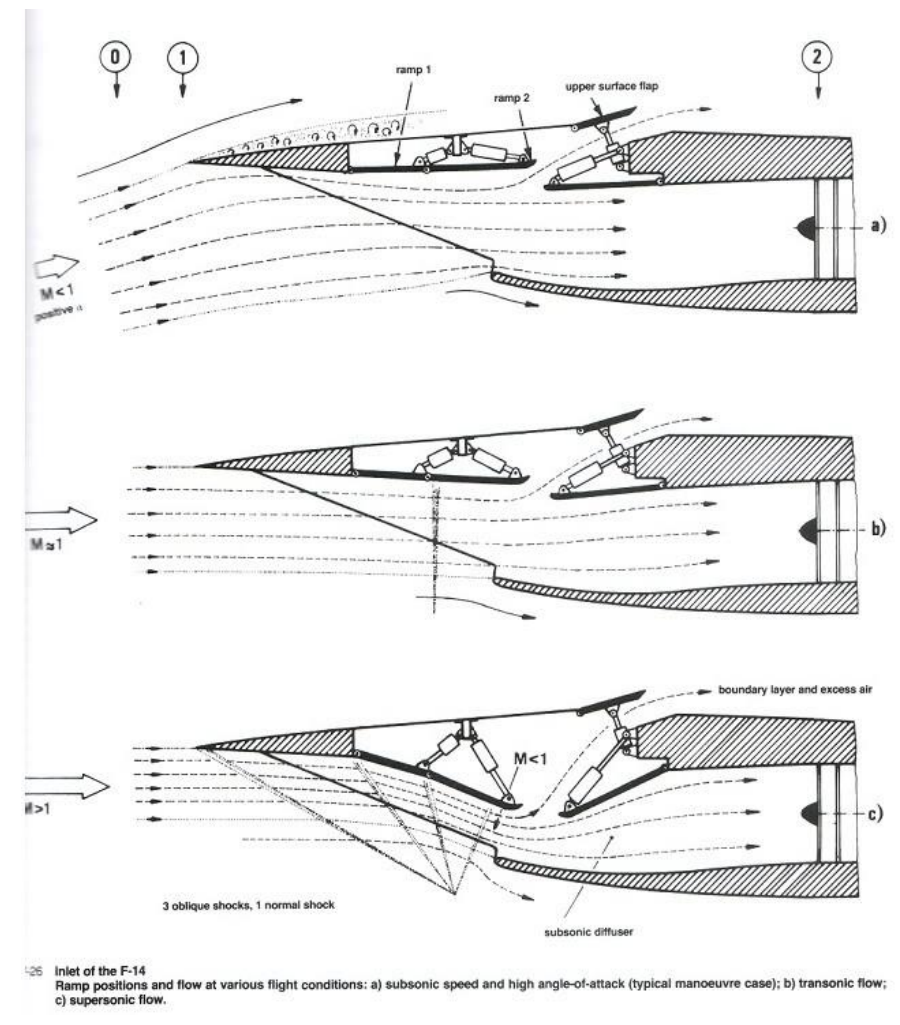

Figure 1: Two-dimensional Ramp Intakes at Different Mach Number

https://aviation.stackexchange.com/questions/13096/what-are-the-merits-of-an-inlet-cone-spikecenterbody-vs-a-2d-inlet

(Aviation Stack Exchange, 2015) 


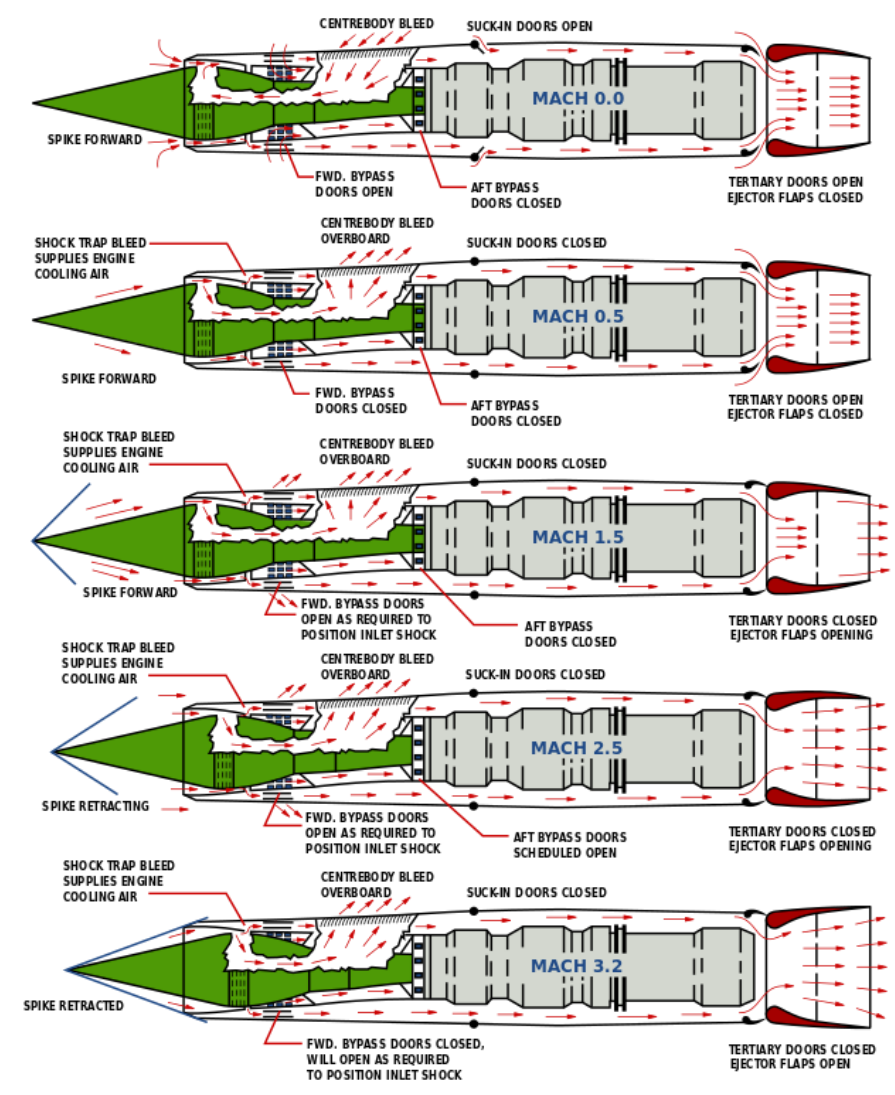

Figure 2: Spike Intakes

\section{https://en.wikipedia.org/wiki/File:SR71_J58_Engine_Airflow_Patterns.svg}

(Free Encyclopedia, n.d.)

To overcome these disadvantages for both spike intake and 2D ramp intake, many researchers have been interested in a promising inlet for high-speed engines. In the late twentycentury, an interest in using 3D air intake appeared attractive for inlets design due to a possibility of obtaining high compression levels of external flow over the inlet ramp with high total pressure recovery. A number of those three-dimensionally curved inlets with circular or elliptical throats were designed and tested in the 1960 s by Hartill ${ }^{1}$, Kutshenreuter ${ }^{2}$ and Kiersey and Snow ${ }^{3}$. To reduce structural complexity, it is preferred for the inlet to have a fixed geometry. These inlets 
showed good performance in the wind tunnel experiments, however, it was difficult to predict the performance with computational tools at that time due to its immaturity. In the mid-1990s, combined numerical and experimental investigation of 3D inlet for scramjet was conducted by Holland ${ }^{4}$. It is reported that nature and structure of the flow interactions inside an inlet in this article.

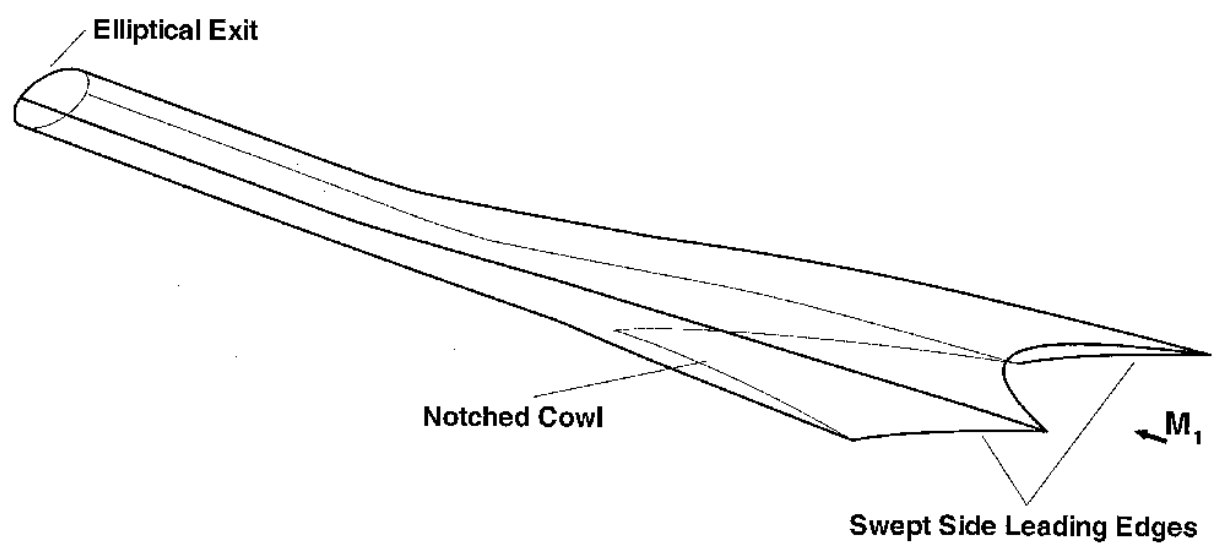

Figure 3: Pictorial View of Three-dimensional Inlet (Smart, 1999) ${ }^{5}$

This work will not go into much detail on the computational investigation, rather would like to highlight experimental measurements. Unfortunately, there are few reports showing such investigation in which focuses on flow interaction taking place around the entrance of threedimensionally curved inlets while a computational investigation was conducted. Further understandings of turbulent flow entering the inlet of high-speed engines are needed for the improvement of the performance. The current project aims at the investigation of shock structures and density gradients caused by strong change in flow characteristics. Note that, to reduce 
measurements difficulty, the problem statement is simplified in this study and a cone spike mounted in supersonic wind tunnel facility is utilized. The spike is axisymmetric, yet the shock structure generated around it is three-dimensional and sufficiently complicated to simulate the inlet of high-speed engines. To investigate the structure and density gradient, they are visualized using SLFF and 3D models are then reconstructed in MATLAB. In this report, the technique is also introduced and described as a novel and promising tool for flow visualization. Expected results from this project are obtaining quantitative measurements that reveal the interaction between freestream flow and high-pressure region in compressible flow. 


\section{CHAPTER TWO: LITERATURE REVIEW}

When a flow moves faster than the local speed of sound, a shock wave is generated to decrease the velocity. This process is no longer isentropic as is different from flows without shock waves and it is characterized by an abrupt change in flow properties such as pressure, temperature and density of the medium. When considering the density change in the flow, the refractive index also changes according to the Gladstone-Dale relation shown below.

$$
\frac{n-1}{\rho}=k
$$

In this equation $n$ is the index of refraction, $\rho$ the density of the flow and $k$ shows a constant value. This relation was described by J. H. Gladstone and T. P. Dale ${ }^{6}$ and indicates that these two properties are proportional to each other. From this correlation, in the 1660s it was proposed that flow with a violent change in density could be visualized based on the deflection of light by a refractive index gradient. This phenomenon was first observed by R. Hooke ${ }^{7}$ and named as schlieren. Later the conventional schlieren system was developed mainly by A. Toepler ${ }^{8}$ and introduced as flow visualization technique to come increasingly into use for the analysis of airflow. It is required for the system to use some optical instruments such as concave mirrors (schlieren mirrors), a light source, a knife-edge and an imaging screen. The light rays generated by the light source reflect back at a concave mirror to illuminate the flow field within the test section. Some of those rays are deflected by the refractive index caused by the density gradients while passing through the field and the whole light hit another mirror. The secondary shadow pattern that creates 
a strong contrast between deflected and undeflected light is cast on the imaging screen by the knife-edge since undisturbed rays are partially blocked at this point. This shadow pattern represents a light intensity of low-density regions and high-density regions that characterize the flow. Note that the light rays after the first concave lens are ideally parallel. Fig. 4 shows a conventional schlieren system and Fig. 5 a z-type schlieren system. The latter is often utilized for wind tunnel testing 9 .

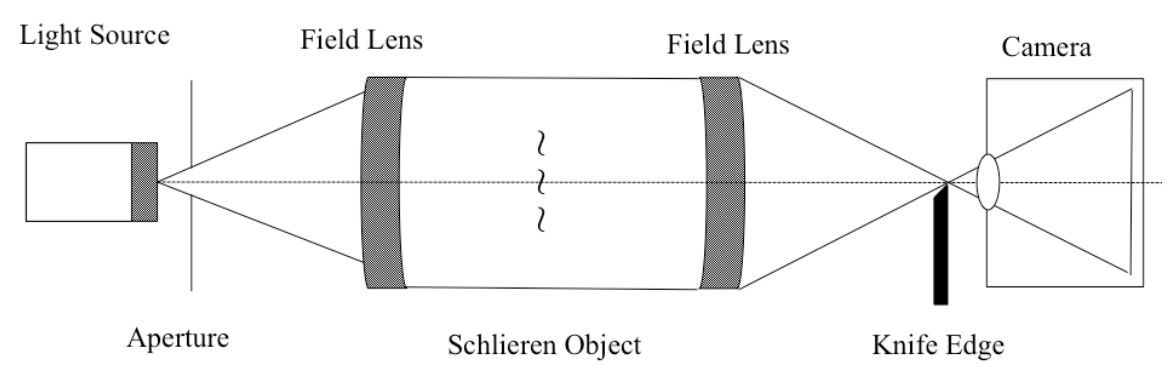

Figure 4: Conventional Schlieren Schematic

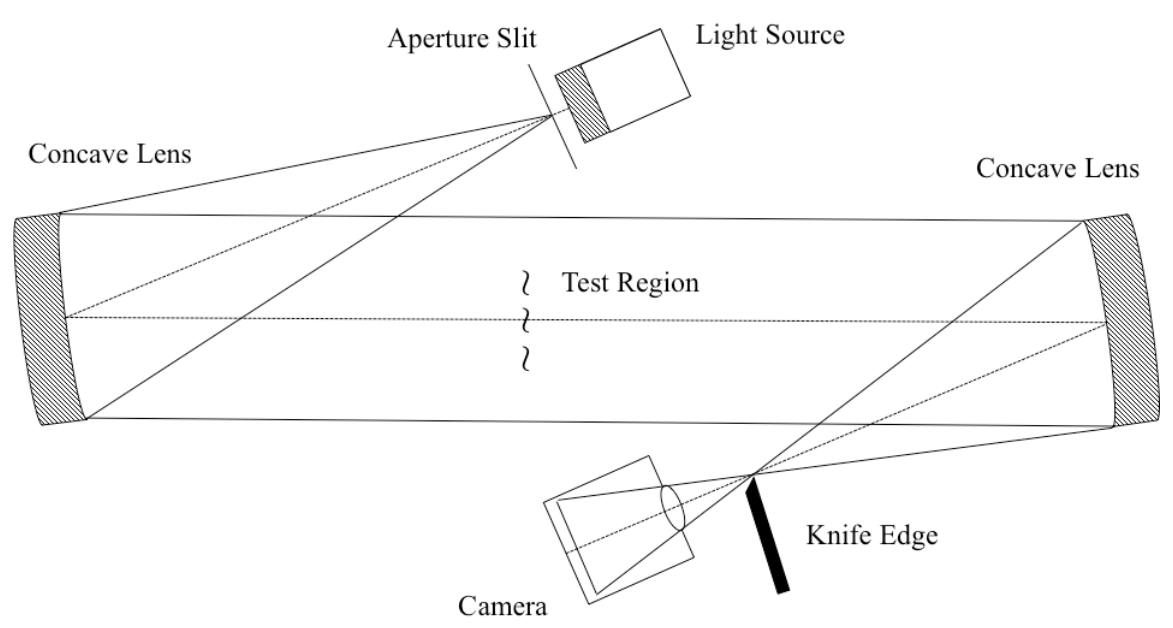

Figure 5: Z-type Schlieren Schematic 
While it has been widely used as one of flow visualization techniques, some limitations were pointed out by G. S. Speak and D. J. Walters ${ }^{10}$. There is briefly one limitation mentioned in their report, that use of schlieren is not recommended for quantitative measurements where small pressure and density changes are involved. Further, it is also concerned that it requires a complex optical setup and yet the visualizable area is limited due to an effective diameter of optical lenses and mirrors. Considering these limitations, improvements on schlieren system have carried out in past few decades. While various visualization techniques have been established, there is one method focused on in this report appeared: Focusing Schlieren. This method was first suggested by A. Kantrowitz and R. L. Trimpi ${ }^{11}$ in the early 1950s, however, it was not spotlighted initially due to its small visualizing area and complexity of optical setup. Hence, improvements on this method were not added until the 1990s. To overcome these disadvantages, L. M. Weinstein ${ }^{12}$ invented and described an improved focusing schlieren device, and nowadays it is widely used for wind tunnel experiments including cryogenic wind tunnel ${ }^{13}$. The improved technique is capable of visualizing density gradients in an arbitrary cross-section with a wide field of view without loss of schlieren sensitivity comparable to the first focusing schlieren. Also, this method can retain the benefits of low cost, easy-to-setup and the capability of making non-intrusive measurements. The system is shown below in Fig. 6. The distances between each optical component are also indicated in this diagram as symbols. As $x-y-z$ coordinate is set and let $x$-axis be perpendicular to the flow direction in this system, it is capable of visualizing x-y planar region. 


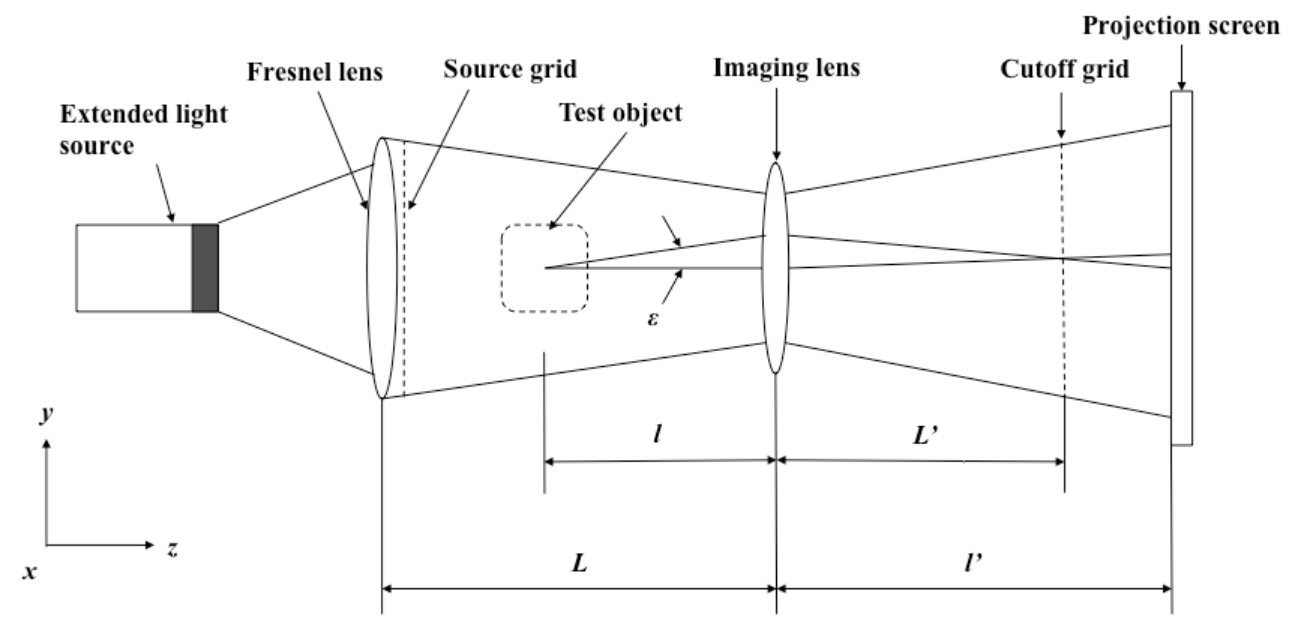

Figure 6: Sharp Focusing Schlieren Optical System

F. S. Alvi, G. S. Settles and L. M. Weinstein ${ }^{14}$ combined such a focusing schlieren system with an optical deflectometer and performed initial benchtop experiments. In this system, the viewing screen was replaced with a clear acrylic flat with fiber optic sensors. These sensors are positioned in the image plane and capable of sensing fluctuations in light intensity in the schlieren image due to turbulent structures in the flow field. Experiments were conducted with the lowspeed, axisymmetric $\mathrm{CO}_{2}$ jet flowing into the still air, which produces large-scale turbulent structures. Carbon dioxide was used in this report since it produces strong density gradients in the process of mixing with ambient air even at low speeds. The jet was initially located at such a position to have its axis perpendicular to the optical axis and the top-center and the bottom-center edges of the axisymmetric shear layer, meaning the jet centerline, were in the plane of best focus. As a result, they successfully observed the Kelvin-Helmholtz vortices in the mixing layer. Additionally, they demonstrated that the unique sharp-focusing effect as the focal plane was dislocated from the centerline of the shear layer. The images of the vortices structures were rapidly 
blurred at displacements of $\pm 2 \mathrm{~mm}$ in the $\mathrm{x}$-axis, which is perpendicular to the flow direction. At $x= \pm 8 \mathrm{~mm}$, the structures completely disappeared and it was indicated that any structures within $4 \mathrm{~mm}$-wide focal volume would be in sharp focus on the screen.
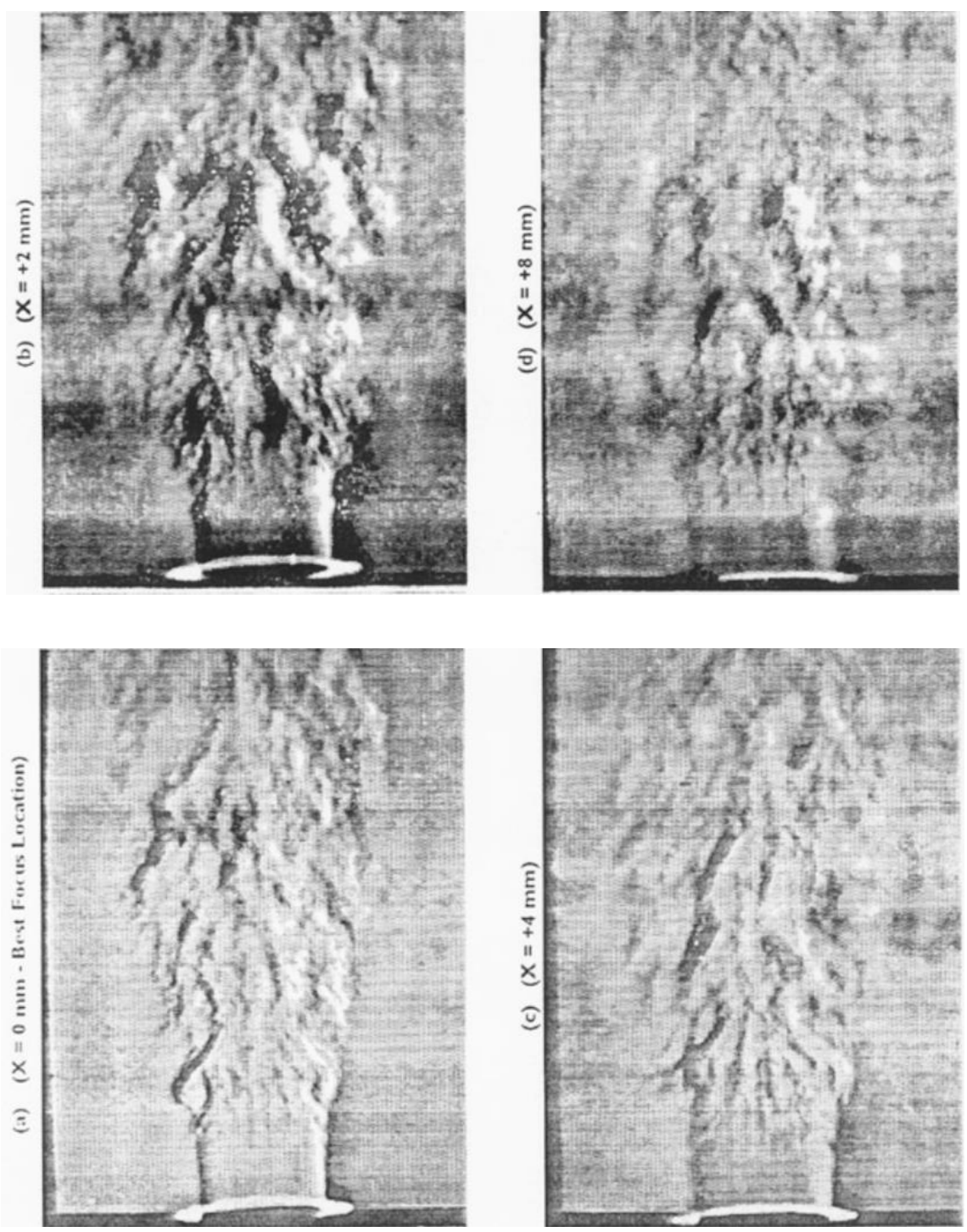

Figure 7: Focused-Schlieren Images of $\mathrm{CO}_{2}$ Jet at Four Axial Locations (Positive X Directions) (F. Alvi, 1993) $)^{13}$ 

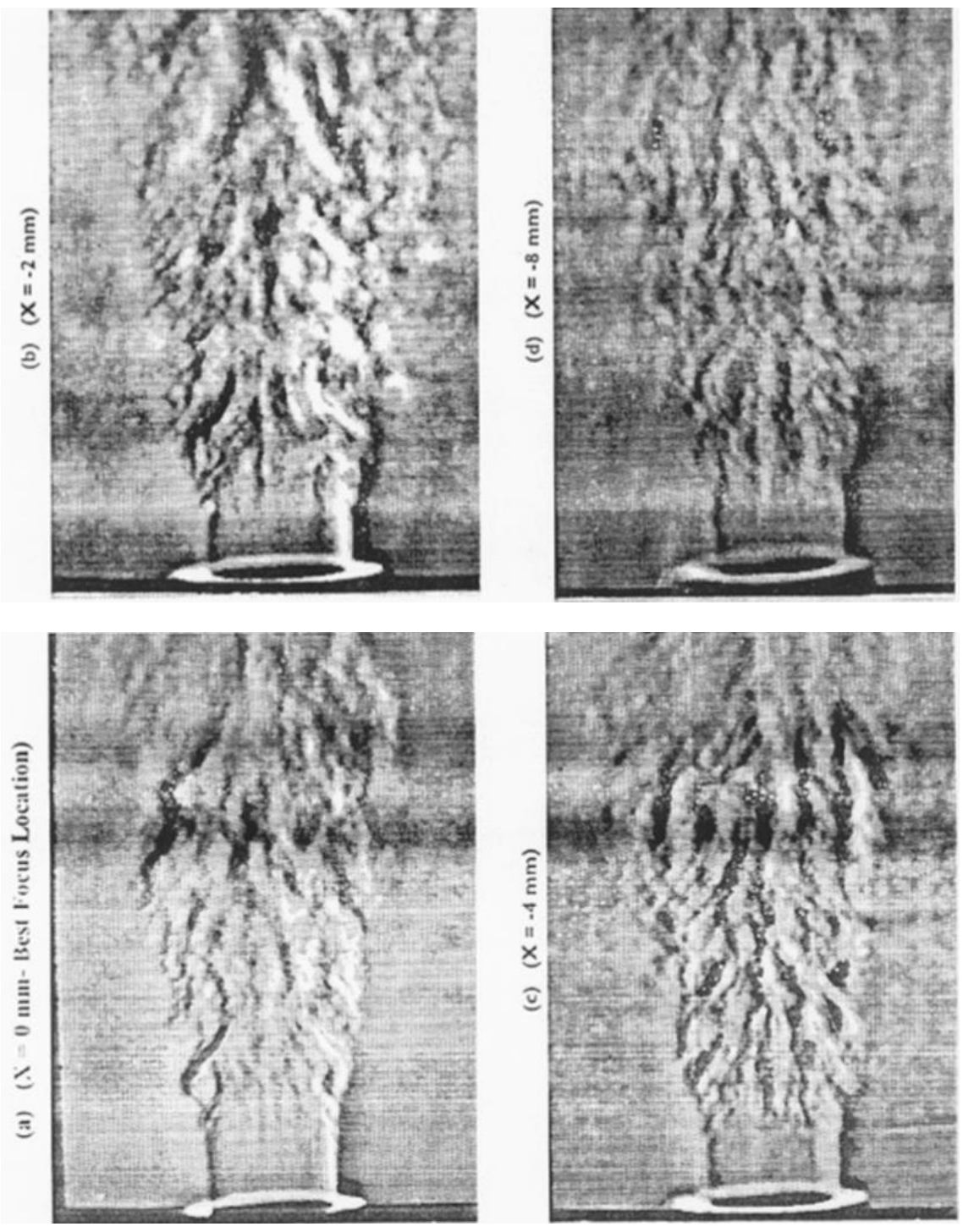

Figure 8: Focused-Schlieren Images of $\mathrm{CO}_{2}$ Jet at Four Axial Locations (Negative X Directions) (F. Alvi, 1993) $)^{13}$

Also, a photodetector was used in this work to investigate quantitatively the depth-of-focus. This was determined by examining the power spectra of the optical data taken in this experiment. When the centerline of the jet sits in the best focus plane, the spectra of the fluctuations indicated a peak as being indicative of the Kelvin-Helmholtz vortices around specific frequency. The peak in 
spectrum disappeared completely by $x=-2 \mathrm{~mm}$ to $+3 \mathrm{~mm}$, thus it is assumed that the instrument is merely sensitive in a planar region of $4-5 \mathrm{~mm}$ approximately in width. Hence, the instrument carried in the study demonstrated quantitatively and qualitatively that highly localized turbulent measurements are possible.

In the recent past, M. Kashitani and Y. Yamaguchi ${ }^{15}$ employed the focusing schlieren system to investigate the aerodynamic characteristics of a double wedge airfoil. In this study, a flow field around the airfoil mounted in a gas-driven shock tube was visualized and compared with the one measured using the conventional schlieren. The shock wave profile taken by the focusing schlieren is shown below in Fig. 9.

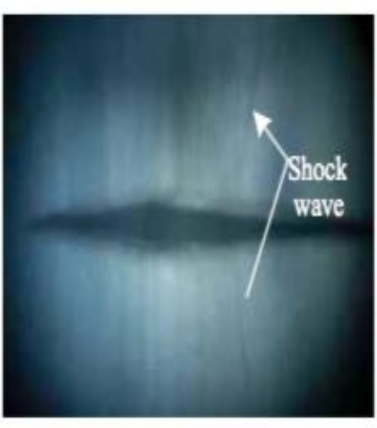

(a) $l=170 \mathrm{~mm}, s=30 \mathrm{~mm}$

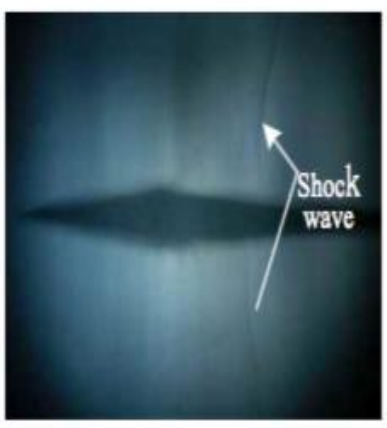

(b) $l=155 \mathrm{~mm}, s=15 \mathrm{~mm}$

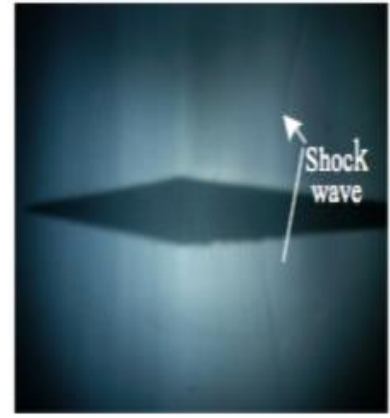

(c) $l=145 \mathrm{~mm}, s=5 \mathrm{~mm}$

Figure 9: Shock Wave Profiles at Different Focal Location

(Yamaguchi, 2006) $)^{15}$ 


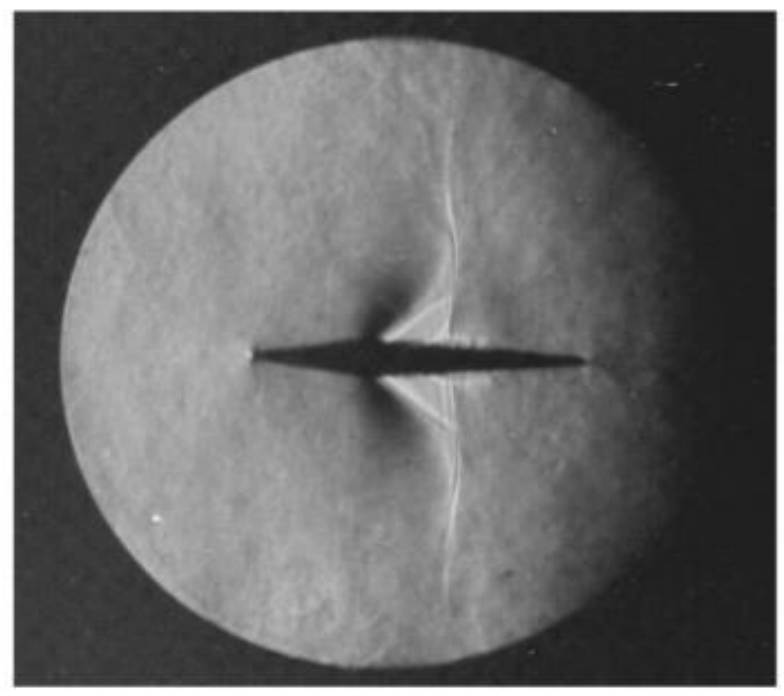

Figure 10: Shock Wave Profile with the Conventional Schlieren Method (Yamaguchi, 2006) $)^{15}$

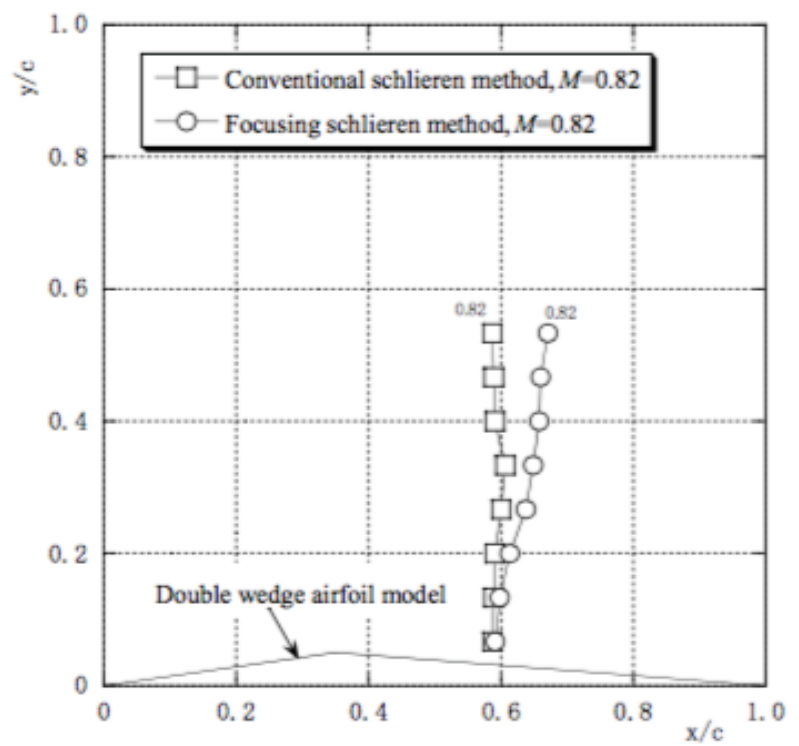

Figure 11: Shock Wave Profile with the Conventional and the Focusing Schlieren Method (Yamaguchi, 2006) $^{15}$ 
The best-of-focus point is located in the middle of the span of the airfoil and exposed in Mach 0.82 with no angle of attack. The shock profile from both Fig. 9 and Fig. 10 were calculated and the location of shock is indicated in Fig. 11. The symbol $c$ represents the length of the chord and used for characterization of the shock location. As can be seen, the foot of shock waves with the focusing and one with the conventional schlieren almost agree while the difference in the profile can be observed as it leaves from the airfoil surface. This is considered as one of the effects caused by the boundary layer development along the wall/airfoil surface. Additionally, they conducted experiments with various Mach number on the airfoil to investigate an effect of Mach number in detail and successfully observed the flow field around the model.

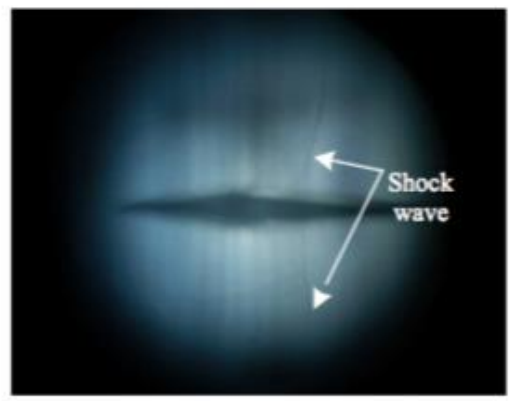

(a) $M=0.80, R e=3.11 \times 10^{5}$

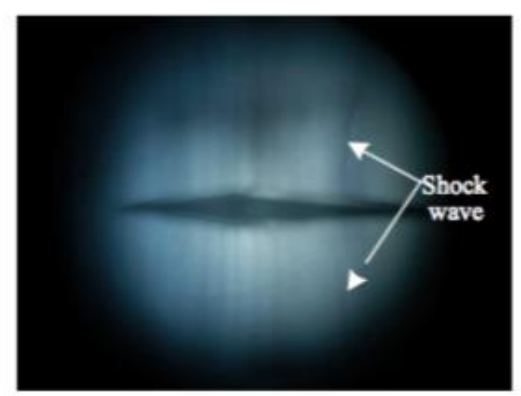

(b) $M=0.84, R e=3.07 \times 10^{5}$

Figure 12: Flow Visualization around the Double Wedge Airfoil Model

(Yamaguchi, 2006) $^{15}$ 


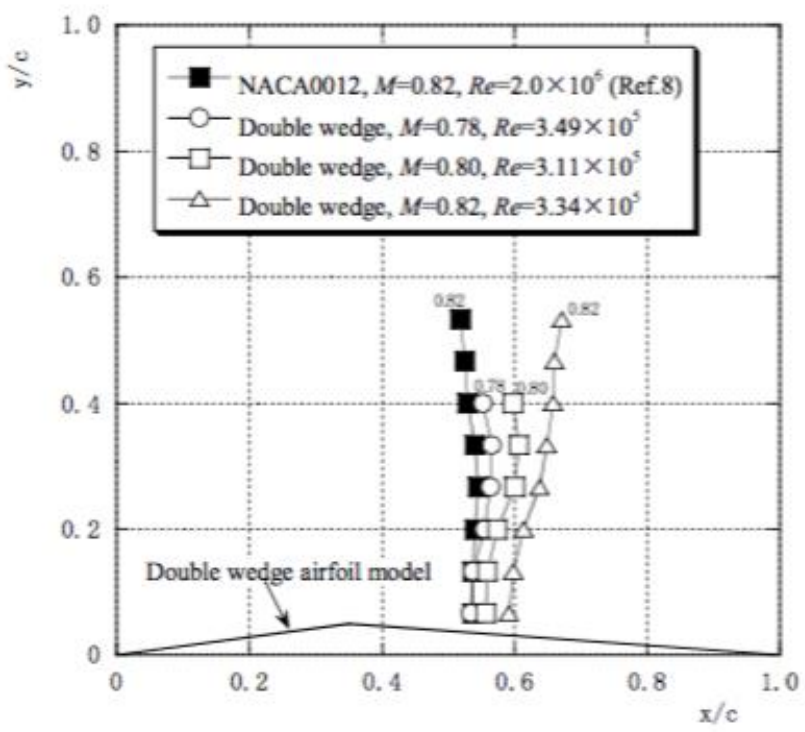

Figure 13: Shock Wave Profile on the Double Wedge Airfoil Model $\left(\alpha=0^{\circ}\right)$ $(\text { Yamaguchi, 2006) })^{15},\left(\right.$ M. Kashitani, 2003) ${ }^{17}$

The symbols $(\circ, \square$ and $\Delta)$ used in Fig. 13 denote respectively the shock wave profile at different Mach numbers. $\square$ stands for the profile of the NACA0012 airfoil model and experiments on this model were carried out with the conventional schlieren by M. Kashitani, Y. Yamaguchi and Y. Nakao $^{17}$. According to the profile results, the location of the shock waves moves to the downstream as Mach number increases ${ }^{18}$. They clarified that a sharp focusing schlieren method is a promising tool for flow visualization by measuring the shock wave profile generated on an airfoil model.

Moreover, in 2015, a focusing schlieren method was carried out for measurements of the volumetric expansion of fuel jet and anchored bifurcated shock wave induced within a dual-mode scramjet ${ }^{19}$. It is remarkable since it was the first time focusing schlieren measurements have been successfully reported for a scramjet. Due to difficulties that the conventional schlieren essentially 
have, it is not suited to capturing turbulent structures in compressible flows. This is because the highly heated flow in a combustion chamber changes the local refractive index of the window glass, resulting in that thermal distortions affect schlieren imaging. Unlike the conventional technique, focusing schlieren shows the potential of reduced sensitivity to capture clear flow structures even during a combustion process. The reduced sensitivity is responsible for the potential ability of the technique to capture an arbitrary planar region within a test section. The experiments were executed in an electrically heated, continuous-flow wind tunnel at the University of Virginia Supersonic Combustion Facility (UVaSCF) that provided clean air simulating Mach 5 flight enthalpy with a stagnation temperature of $1200 \mathrm{~K}$ and a stagnation pressure of $300 \mathrm{kPa}$. A schematic of a dual-mode scramjet is shown below. The flow path is directly connected to the Mach 2 facility nozzle.

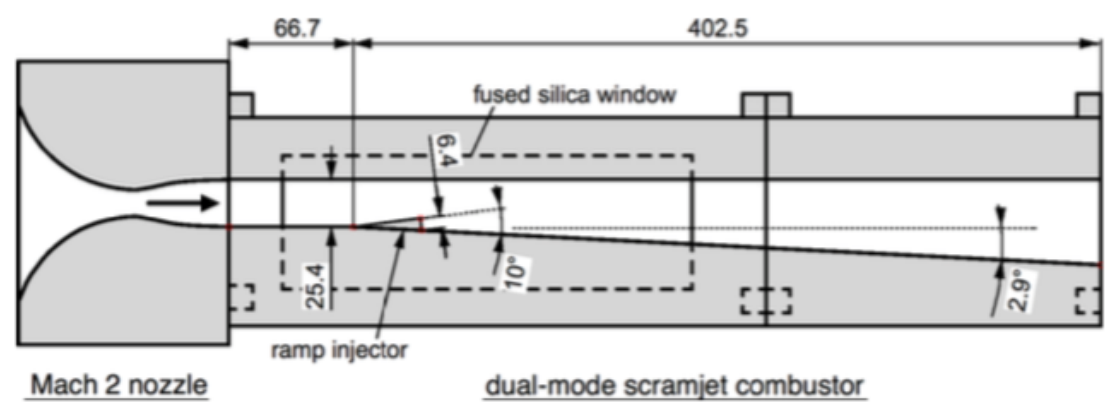

Figure 14: Schematic View of the Direct-Connect Dual-Mode Scramjet with a Ramp Injector $\left(\mathrm{K}\right.$. Toshinori, 2015) ${ }^{19}$ 
Since the static temperature in the combustor was below the auto-ignition temperature, measurements were performed for fuel-air mixing as well as fuel-air reacting. A hydrogen-oxygen detonation-driven igniter was applied to ignition. The fuel flow rate was increased to an overall equivalence ratio of 0.2 for successful ignition. After igniting the mixture, the equivalence ratio was decreased to 0.17 . The combustion process with the mixture was even sustainable with such the ratio once ignition occurred. Flow visualization measurement was conducted for both fuel-air reacting and the ignition process. Also, it was conducted without fuel injection for various stagnation temperatures in the chamber during warming up the facility to investigate the effect of the temperature, which has a strong relationship with window distortion. Figures shown below represent typical images for cold flows. The downstream end of the ramp lies in the lower left corner in these images.

(a)

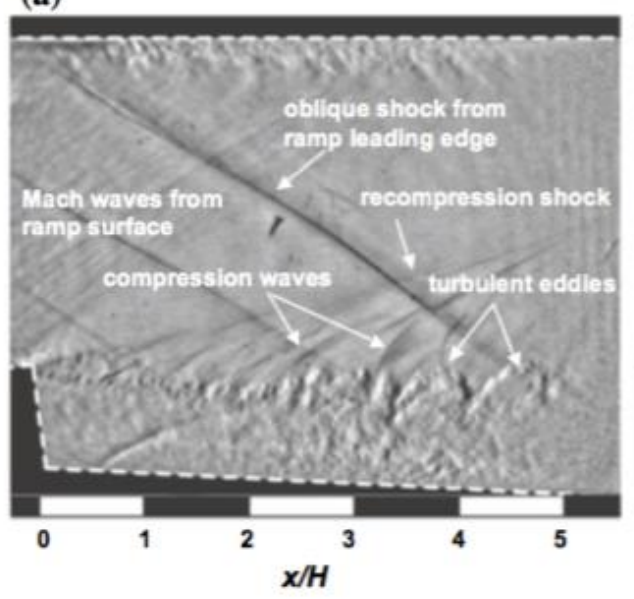

(b)

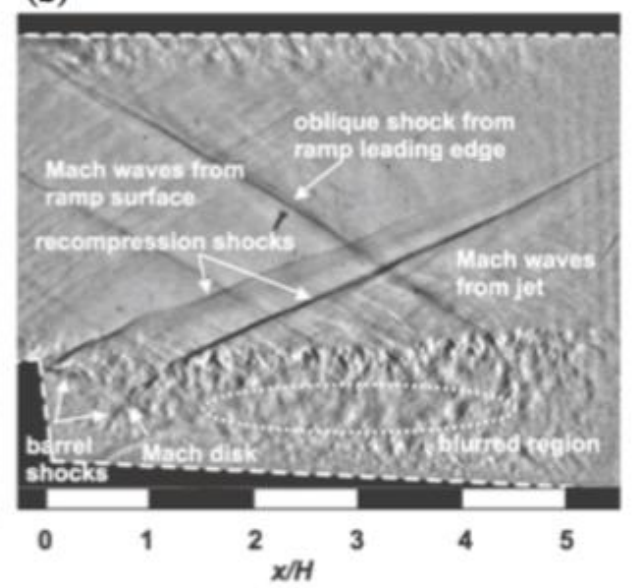

Figure 15: Typical Focusing-Schlieren Images under Cold Flow Condition: a fuel off and b fuel on

$\left(\right.$ K. Toshinori, 2015) ${ }^{19}$ 
Here, $H$ denotes ramp injector-surface-normal height of $6.35 \mathrm{~mm}$ and is used to normalize the spatial variables. For the case of fuel off, it can be observed that large turbulent eddies were generated at $x / H>3$ in the wake and compression waves were emitted from these structures. Many of these were merged with each other and formed a recompression wave after the end of the ramp. Additionally, due to the smaller velocity of the convection than that of the mainstream, the recompression wave at around $x / H \sim 4$ look more like a bow shock. For the case of fuel on, two shock waves were captured in the mainstream caused by the fuel injection: one is from the trailing edge of the ramp, the other from the Mach disk position of the first shock cell in the jet. As can be seen in Fig. 15(b), some compression waves were generated and associated with the fuel injection. The shape of these waves seems spherical and different from that of Fig. 15(a). The images shown below were taken during the fuel-air ignition and reacting process. In Fig. 16, (a) is an image taken before ignition and (b) after ignition. The equivalence ratio is fixed at 0.2 for both of cases. Fig. 17 represents a focusing schlieren image taken during fuel-air mixing with the equivalence ratio of 0.17 . 
(a)

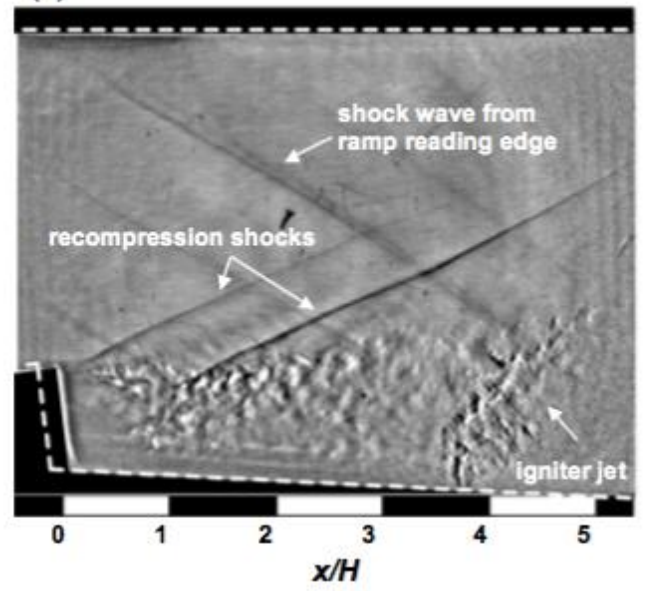

(b)

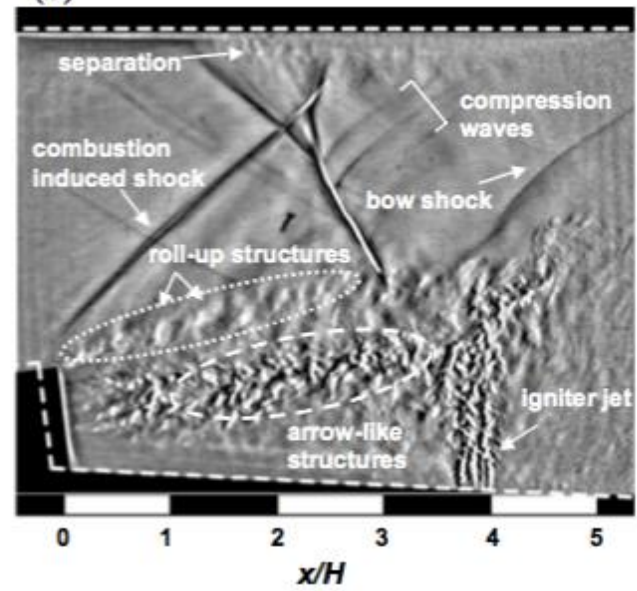

Figure 16: Typical Focusing Schlieren Images for the Case with Igniter On: a before flow path ignition and $\mathbf{b}$ after flow path ignition

$\left(\mathrm{K}\right.$. Toshinori, 2015) ${ }^{19}$

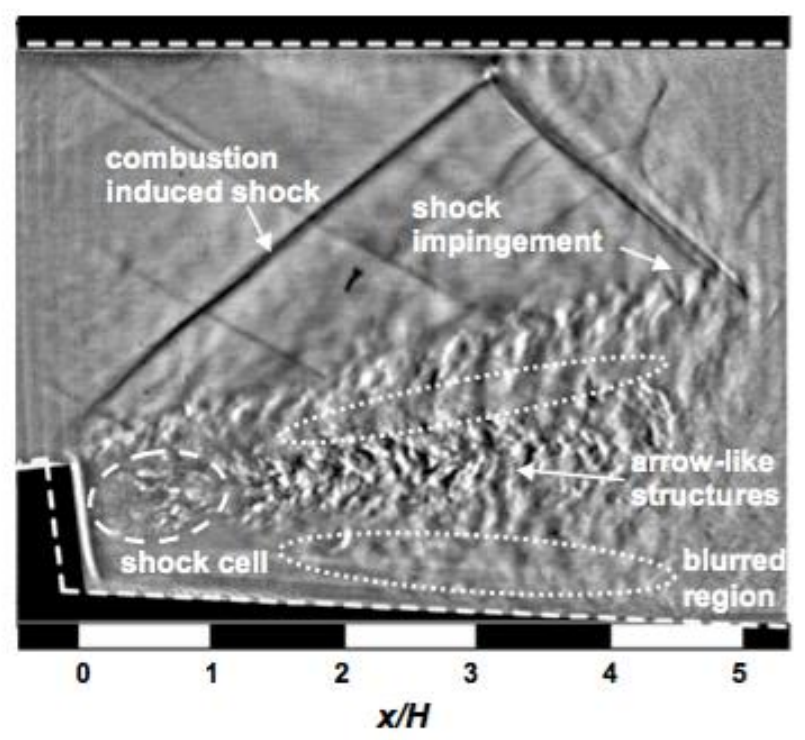

Figure 17: Typical Focusing-Schlieren Image for the Case of Fuel-Air Reacting $\left(\right.$ K. Toshinori, 2015) ${ }^{19}$ 
Summarizing the previous work on the topic, all previous studies described in this chapter lead to the fact that focusing schlieren is capable of visualizing and focusing on a 2D planar region within the test section. Thus it is possible to capture an arbitrary cross section with reduced noise due to its focusing effect. Moreover, the system would not require complex setup, rather simpler optical construction. It is also possible to improve the focusing effect and achieve a smaller depth of focus by combining focusing schlieren and other instruments such as deflectometer as we saw in this chapter ${ }^{14}$. Described in this study are a newly introduced technique of a focusing schlieren and a measurement of supersonic shock structures around an obstacle utilizing the technique. 


\section{CHAPTER THREE: EXPERIMENTAL METHODS}

As we see in Chapter 2, a general focusing schlieren system is consisted of few optical instruments: an extended light source used to illuminate back of a source grid, a Fresnel lens collecting light rays form the source so as to spread them on the cutoff grid, a source grid which has black and transparent stripes evenly and closely spaced to provide multiple light rays, an imaging lens focusing to form an image on a projection screen, a cutoff grid equivalent to a knifeedge for the conventional schlieren systems to cut down some portions of collected light rays, and an imaging screen ${ }^{20}$. Note here that the cutoff grid is ideally a perfect negative image of the source grid. The multiple bright bands leaving from the source grid act as individual schlieren image and they illuminate a part of the imaging plane while the entire source grid forms the effective light structure of focusing schlieren. This is the basic principle that enables us to take a 2D cross-section. Each black band in the cutoff grid, on the other hand, corresponds to individual knife-edge in the conventional schlieren. Adjusting the position of the cutoff grid minutely allows us varying cutoff percentage from $0 \%$ to $100 \%$. For an undeflected light ray by the change of refractive index, it passes through the cutoff grid to create a bright region on the screen. On the other hand, a deflected ray is blocked by the dark band in the grid to create a shadow.

Unlike the conventional schlieren, that intrusively measure the line-of-sight integrated density change, focusing schlieren has some remarkable advantages. As mentioned before, it is capable of focusing on a 2D planar region with reduced noise from the ambient and measuring with large fields of view at low cost. Additionally, less space is required for either side of test section due to its simple setup. In this study, a newly developed technique based upon focusing 
schlieren and inverse principle of tomography or light field camera is introduced. This technique potentially has a possibility of achieving a smaller depth of focus and less noise from the ambient, yet it remains simple to construct and captures large field of view. To describe details, it is now considered below the concept of light field and a related topic of a plenoptic camera.

Light field or plenoptic function is a spatial and vector description that dictates the amount of light propagating in every direction in space. It was first introduced by M. Faraday ${ }^{21}$ in 1846 and described more by A. Gershun ${ }^{22}$ in 1936. It was found that the amount of light reaching points in space varies from place to place and can be characterized using calculus and geometrical analysis in his paper. He also defined the light field at each point in space as a $5 \mathrm{D}$ function. If the concept is restricted to geometric optics, that is spatially incoherent illumination, and to objects larger than the wavelength of light, the fundamental light carrier is then a ray. Since coordinates and angles can parameterize rays in the spatial domain as Fig. 18 shows below, it can be expressed in $5 \mathrm{D}$ function $^{23}$. If the ambient air is assumed to be transparent and the rays of interest are furthermore limited to the light leaving one point on the convex hull of a bounded object, then the value of the plenoptic function needs to be represented merely along some surface that surrounds the object since the radiance along a ray through empty space remains constant. Thus, the plenoptic function can be reduced to 4D function $24,25,26,27$. 


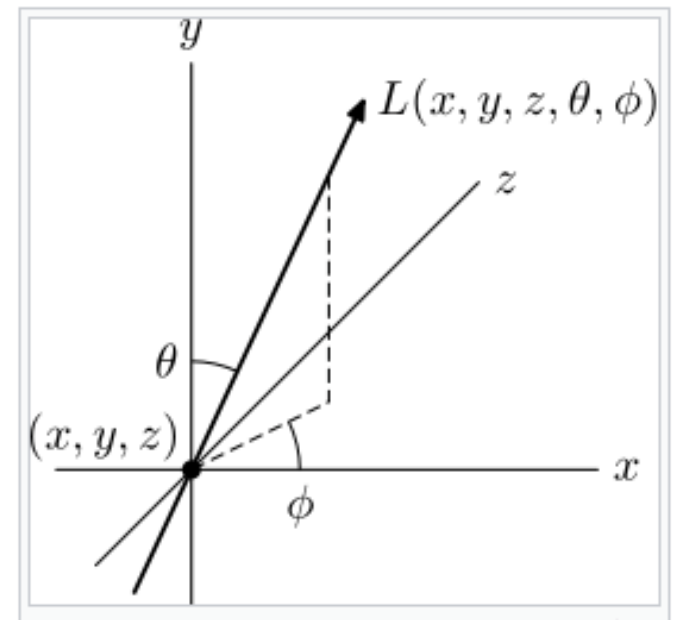

Figure 18: Parameterizing a Ray by Position $(x, y, z)$ and Direction $(\theta, \varphi)$ $(\text { Levoy, 2006) })^{23}$

The principle of light field has been generally used for computer graphics and vision computer photography. Since the concept has then been extended to traditional photography, Adelson and Wang proposed the design of a plenoptic camera (also known as light field camera) using a single lens ${ }^{28}$. Ng et al. advanced the camera inserting a micro-lens array between the sensor and main lens of conventional camera ${ }^{29}$. The basic optical configuration comprises a photographic main lens, a micro-lens array and a photo-sensor array as shown in Fig. 19. 


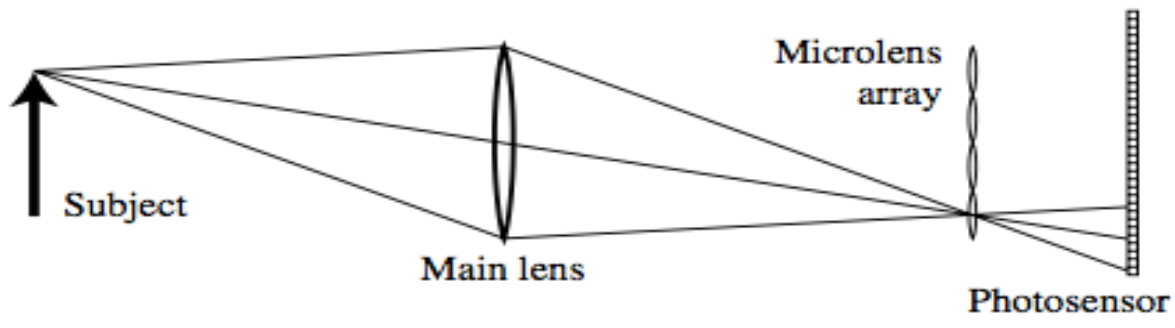

Figure 19: Conceptual Schematic of a Plenoptic Camera

(R. Ng, 2005) $)^{29}$

Light rays from a point on the subject are brought to converge at a point in the focal plane of the micro-lens array. The array separates the rays into sub-images based upon their directions on the photo-sensor sits behind it. Macroscopically, the resulted image is essentially the same as a conventional photograph. Microscopically, however, the sub-images captured by each micro-lens can be seen in the entire image. This whole image that recorded 4D light field function is called light field data and formed by extracting the same pixel under each micro-lens.

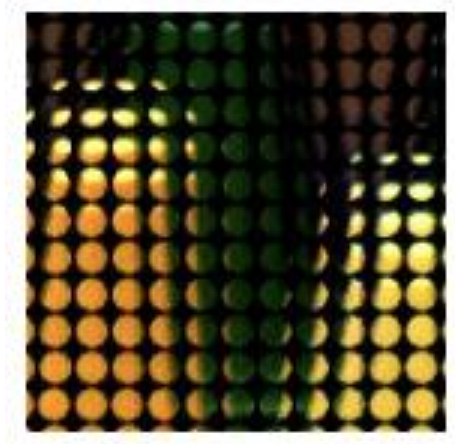

Figure 20: Light Field Data

(R. Ng, 2005) $)^{29}$ 
This data can be characterized by considering the two-plane light field lie between the main lens and the micro-lens, let us denote $L$, where $L(u, v, s, t)$ represents the light traveling along the ray that intersects the main lens at $(u, v)$ and the micro-lens at $(s, t)$. Each extraction corresponds to holding $(u, v)$ fixed while considering all coordinates of $(s, t)$. In other words, at some point in the sub-image corresponds to a specific point in the main lens and they are conjugate along a light ray. Thus, it is possible to virtually modify the shape or position of the aperture by purposely selecting the conjugate coordinates in both lenses. It is important to note here that the limitation of such modification is dependent on the original aperture of the main lens. Additionally, the extracted image is essentially the same as a conventional photograph if taken with that sub-aperture as the lens opening.
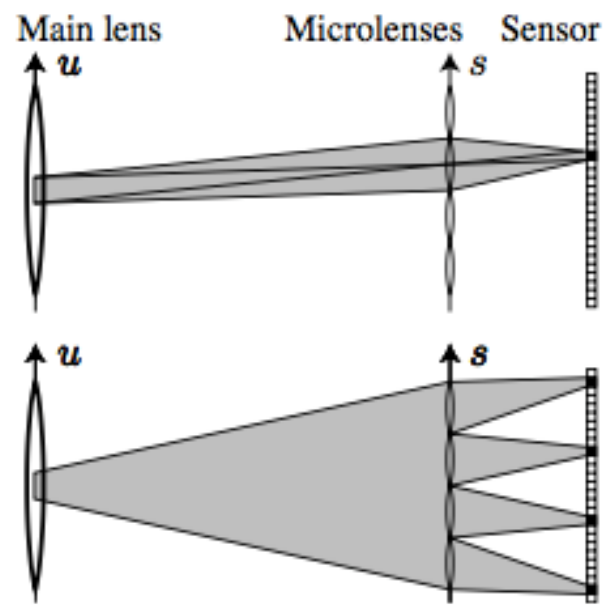

Figure 21: Two-Plane Light Field

(R. Ng, 2005) $)^{29}$ 
The sub-aperture images with parallax can be obtained by modifying the sub-apertures, as shown in Fig. 22. Extracting or adding the sub-images, therefore, allows us to modify the whole depthof-field as a preference.
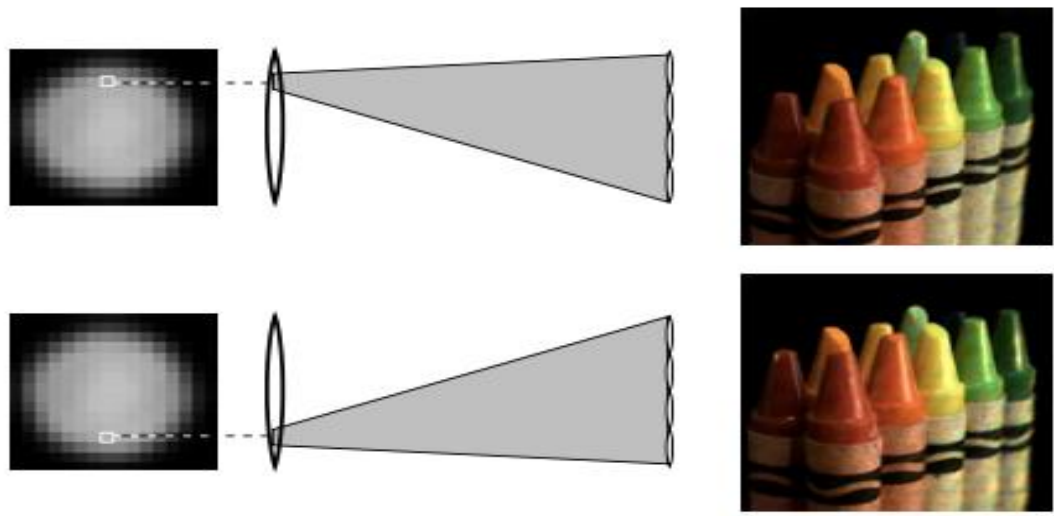

Figure 22: Two Sub-Aperture Photographs with Vertical Parallax

$(\text { R. Ng, 2005) })^{29}$

The plenoptic camera has been explored for the use of flow field velocimetry measurements due to such traits. In the 21st century, Fahringer and Thurow developed volumetric particle image velocimetry with a plenoptic camera ${ }^{30,31,32,33,34}$. Although their attempt resulted successfully, it is pointed out that it is not suited for the use of image tomographic reconstruction due to computational intensiveness and less pixel and spatial resolutions. Multiple camera tomography system, instead, has been the dominant method for three-dimensional PIV, spray and flame imaging. Projections from various angles are taken and processed to reconstruct three- 
dimensional structures. Like plenoptic-reconstruction, this method is limited by pixel resolutions and the number of cameras. Both plenoptic and tomography focus on capturing the complete distribution of light rays on a camera and then reconstruct 3D flow field. The structured light-field focusing schlieren (SLFF), the new method introduced in this study, is based upon focusing schlieren mentioned above and the inverse principle of these techniques. Like both techniques capture images with parallax, SLFF utilizes focusing effect to capture the two-dimensional plane of interest rather than the complete light distribution for non-intrusive flow field measurements.

The schematic of SLFF is shown in Fig. 23. It is consisted of few optical instruments: a cluster of LED light bulbs that each bulb sits in a circle, a source grid to structure the light field, a plano-convex lens to focus light rays down, a cutoff grid to make shadow patterns in images, a capturing camera and a pulse generator that connects the camera and the light source. The cluster of LED bulbs from Integrated Design Tools Inc. (IDT) provides light with $216 \mathrm{~W}$ powered by IDT switching power supply. Each light ray from the light source can be considered as conventional schlieren image with parallax. The light source and the camera are connected to synchronize each other. Thus, it is possible to let the camera sample images at a specific exposure time while the light blinks at some frequency. As a result, the camera can filter the unwanted noise from the ambient. The source grid and the cutoff grid have approximately the same size of $85 \mathrm{~mm}$ width and $100 \mathrm{~mm}$ height. The source grid is responsible for creating semi-parallel light rays for the light field, and the cutoff grid acts as knife-edge for conventional schlieren to partially block the rays to make shadow patterns that indicate density gradient in the image. The Fresnel lens, like the one we see in regular focusing schlieren, is replaced with the plano-convex lens. The focusing schlieren employs the Fresnel lens to take a single light source and spread it on the cutoff grid. In SLFF, 
since the multiple light sources are applied to this system, the lens is no longer needed. This lens has $150 \mathrm{~mm}$ focal length with a F-number of 1.43. A 14bit cooled CCD camera that is manufactured by PCO obtains recorded images. To observe the density field with different focal planes, the depth-of-field is changed evenly by $\pm 1 \mathrm{~mm}$ throughout all testing. Since the estimated depth-offocus is approximately $5 \mathrm{~mm}$ to $6 \mathrm{~mm}$, it is assumed that taking 3 images for both sides is sufficient. More details of the determination of the focal depth are described in the following section. The symbols used in Fig. 23 represent the distance between each optical instrument and the refraction angle as can be seen. These distances are governed by the thin lens formula.

$$
\frac{1}{f}=\frac{1}{o}+\frac{1}{i}
$$

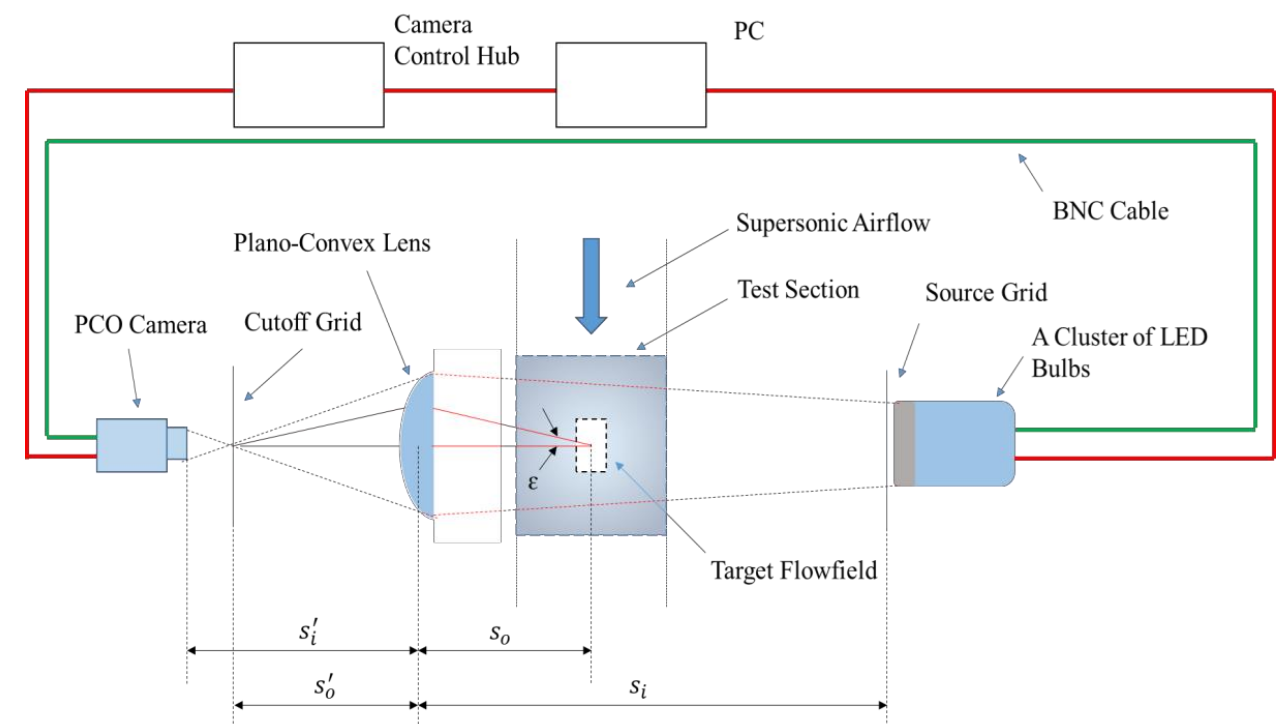

Figure 23: Schematic of SLFF 


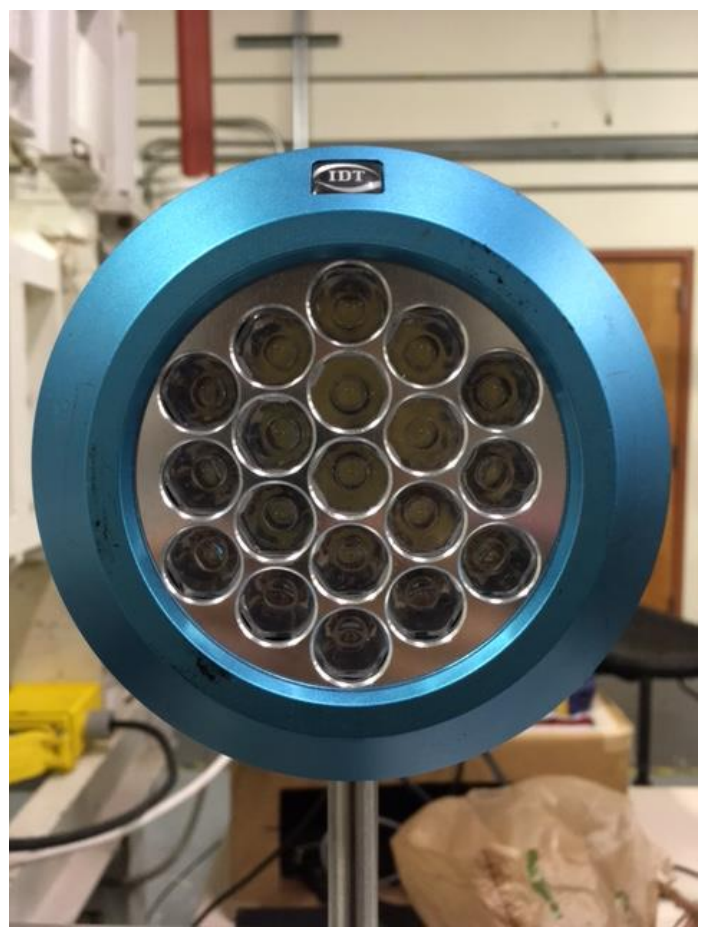

Figure 24: A Cluster of LED Light Bulbs

Individual aperture
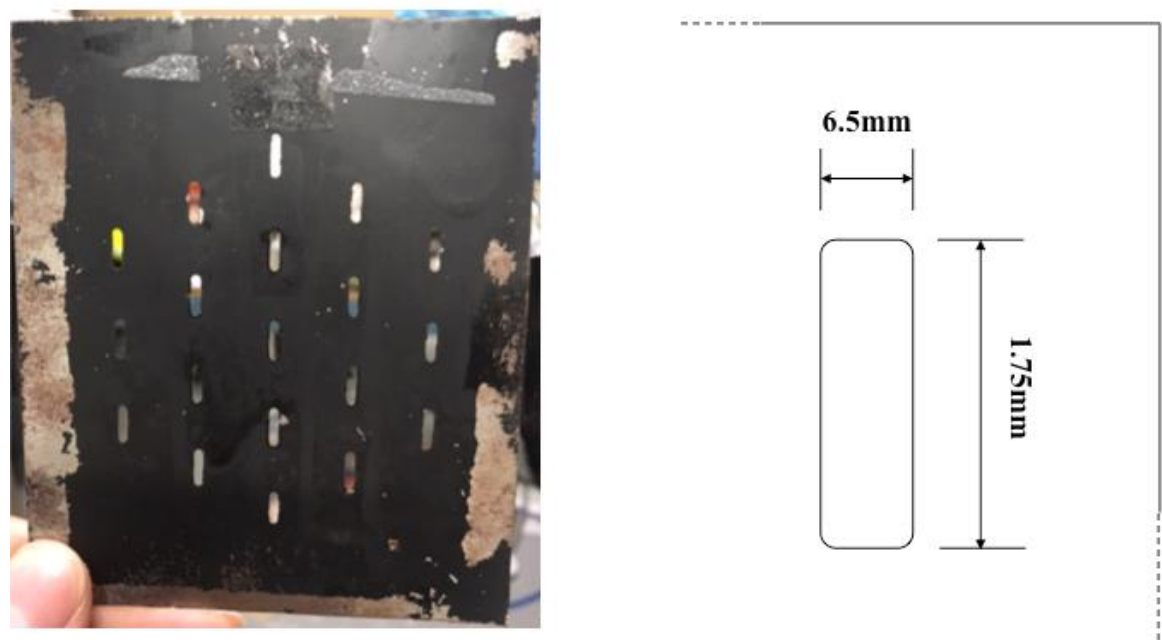

Figure 25: Specification of Source Grid 


\section{Individual aperture}

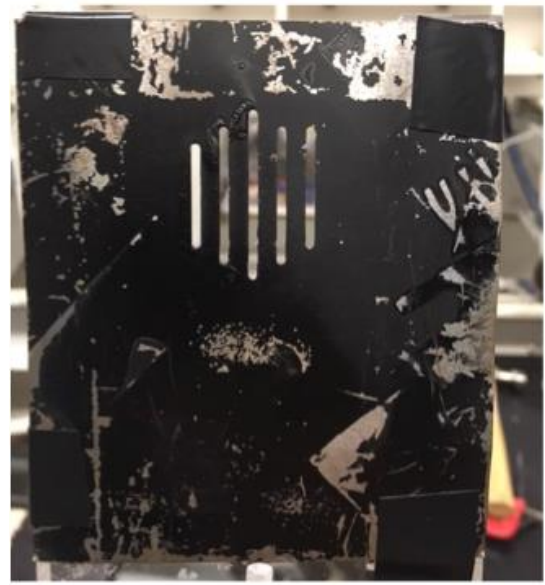

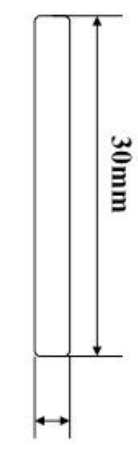

$1.75 \mathrm{~mm}$

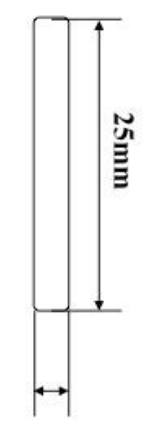

$1.75 \mathrm{~mm}$

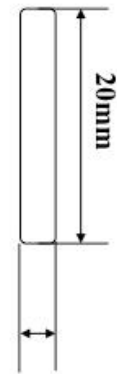

$1.75 \mathrm{~mm}$

Figure 26: Specification of Cutoff Grid

Thus, the distance $s_{o}$ is followed by the distance $s_{i}$ and the lens focal length governs $s_{i}$, and the same for $s_{o}^{\prime}$ and $s_{i}^{\prime}$. Note that the relations of these distances are conjugate for each other and these parameters were experimentally verified. Similarly, the magnification, $m$, produced by the imaging lens can be expressed using these parameters.

$$
m=\frac{s_{o}^{\prime}}{s_{i}}
$$

Using Eq. (2), the magnification can be attained in a simple form as a function of the distances.

$$
m=\frac{1}{\frac{s i}{f}-1}
$$


These instruments are mounted on an optical plate that is placed under the supersonic wind tunnel to capture the entire test section. The foundation of the plate is attached to a stepping motorpowered traverse manufactured by Superior Electronics Corp. It is, therefore possible to translate the whole setup by a millimeter. The translation mechanism is controlled by a remote controller connected directly to the stepper motor.

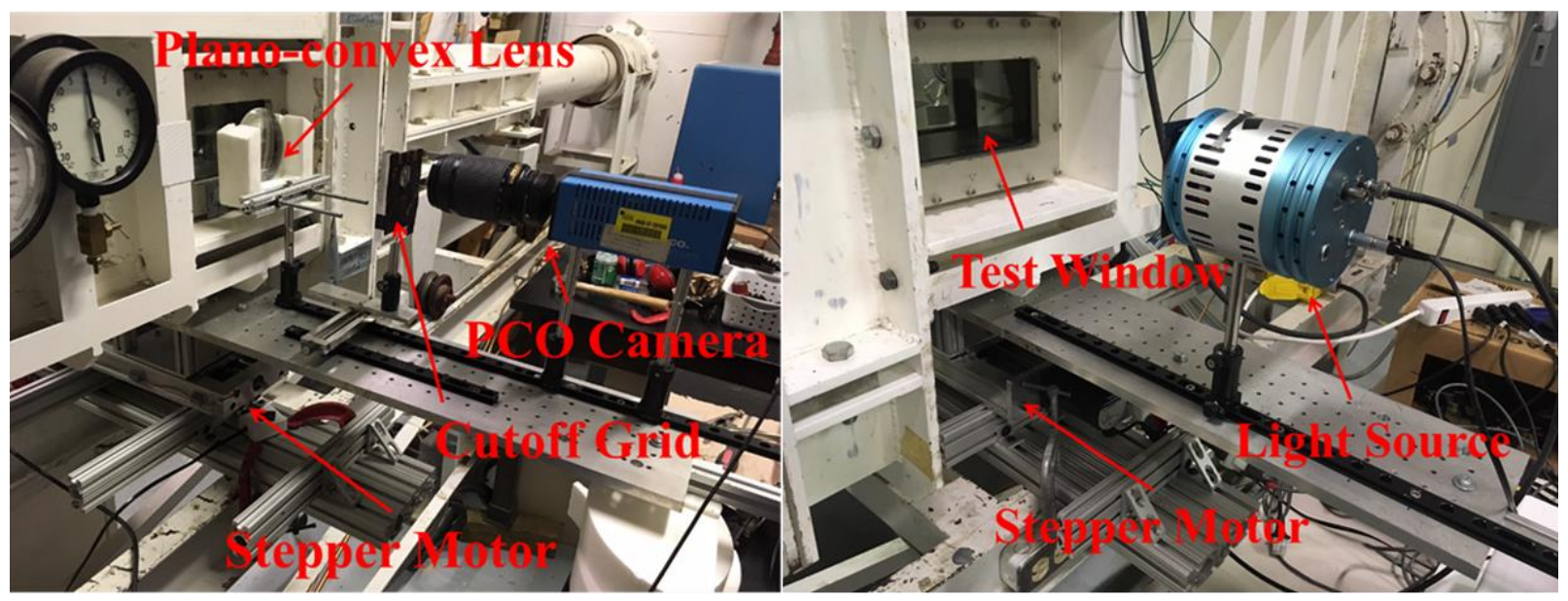

Figure 27: Actual Setup of Current System

In previous work conducted by K. Ahmed and A. Wiley ${ }^{35}$, the generic model of structured light-field focusing system was derived from a set of equations that are developed from the thin lens equation. They introduced and explored some important variables to characterize the structured light-field focusing system. 
Table 1: Summary of Explored Variables

\begin{tabular}{|c|c|}
\hline Parameters & Variables \\
\hline Focusing Lens (Focal Length/Diameter) & $f^{\#}$ \\
\hline Light Sources Offset Distance & OS \\
\hline Number of Light Sources & $N^{\#}$ \\
\hline Depth of Focus & DOF \\
\hline
\end{tabular}

From these various parameters and system settings, a relationship for the DOF of the focusing system was defined. The following figure shows the resulted DOF for each configuration of the optical setup. Different numbers of the LED bulbs, characteristics of the imaging lenses and focal lengths of the camera were tested.

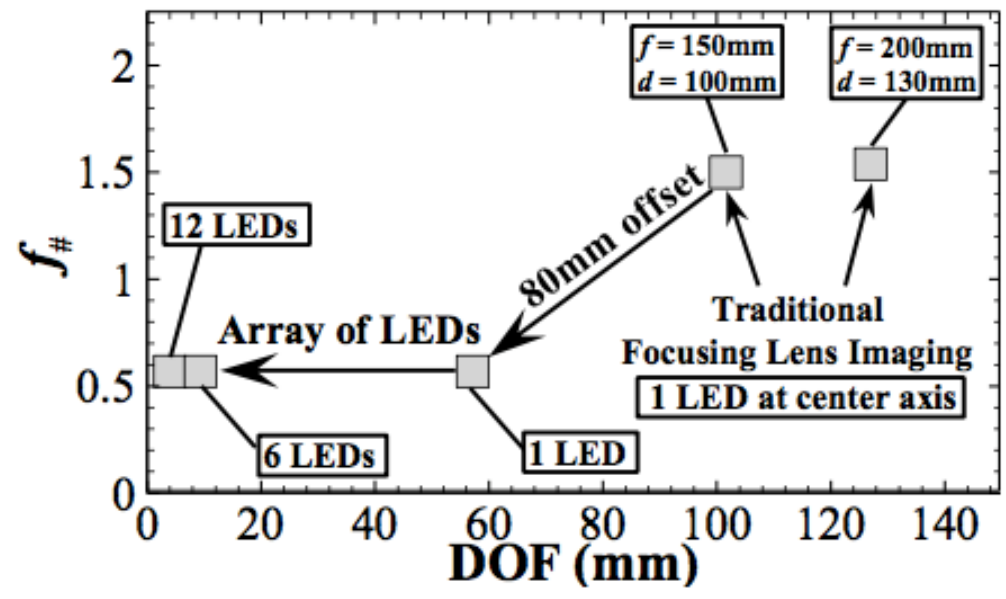

Figure 28: Different Optical Configurations Tested

(Wiley, 2017) $)^{35}$ 
It requires two major parameters to configure the system: the spatial distance of $s_{i}$ and either the desired DOF or the F-number of $f^{\#}$. The fundamental thin lens equation reveals the displacements of each optical instrument as shown above. Thus, the distance between the plane of focus and the imaging lens, $s_{o}$, can be obtained from Eq. (2) when the focal length of the lens is known. These parameters determine the distances of each instrument, however, a narrow DOF that is a significantly critical parameter characterizes the focusing system. Considering a single light source along a center axis in Fig. 30, the DOF can be derived from Eq. (5)-(9). Here, let us denote $c$ the level of defocusing.

$$
\begin{aligned}
& \text { DOF }=\frac{2 I_{1} \frac{f^{2}}{f^{2} c}\left(I_{1}-f\right)}{\frac{f^{4}}{f^{2} c^{2}}-\left(I_{1}-f\right)^{2}} \\
& f^{\#}=\frac{f}{d} \\
& c=\frac{D O F}{I_{1}+\frac{D O F}{2}} \frac{f^{2}}{f^{\#}\left(\left[I_{1}-\frac{D O F}{2}\right]-f\right)} \\
& D_{F}=I_{1}+\frac{D O F}{2} \\
& D_{N}=I_{1}-\frac{D O F}{2}
\end{aligned}
$$


From these relations, the DOF can be further reduced by modifying $I_{1}$ in Eq. (5) since the F-number is a function of lens diameter and focal length that are fixed parameters. Therefore, the F-number can also be reduced by offsetting the light source from the optical center axis. This offset distance is defined as OS in their study and it forms an imaginary lens with a larger diameter, while it has the same focal length. Hence, the F-number is modified using the OS.

$$
f^{\#} \approx \frac{f}{d+(2 O S)}
$$

Also, it was determined a linear relationship between the number of light sources using experimental data as follows.

$$
D O F_{\text {system }} \approx \frac{D O F}{N^{\#}}
$$

Though it is theoretically possible to achieve an infinite thin DOF by infinitely increasing the number of light sources, there is an upper limit for the configuration. The limitation is based upon the geometry of the light source array that each source can be placed in such as ring-shaped in Fig. 24. Further implementations or improvements on the spatial limitation can achieve thinner DOF. 


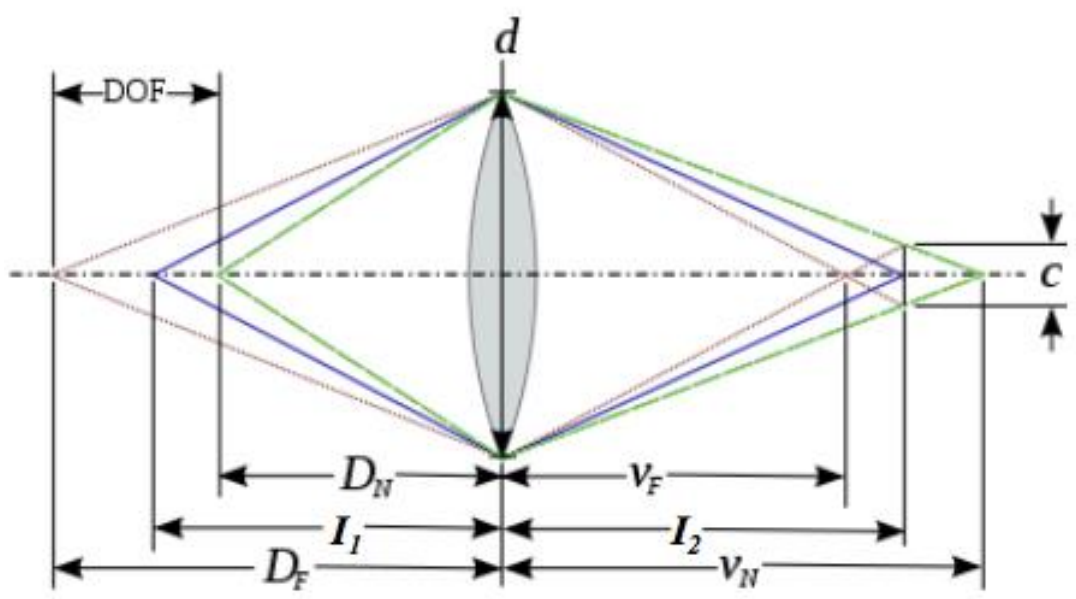

Figure 29: Focusing Lens Parameters

(Wiley, 2017) $)^{35}$

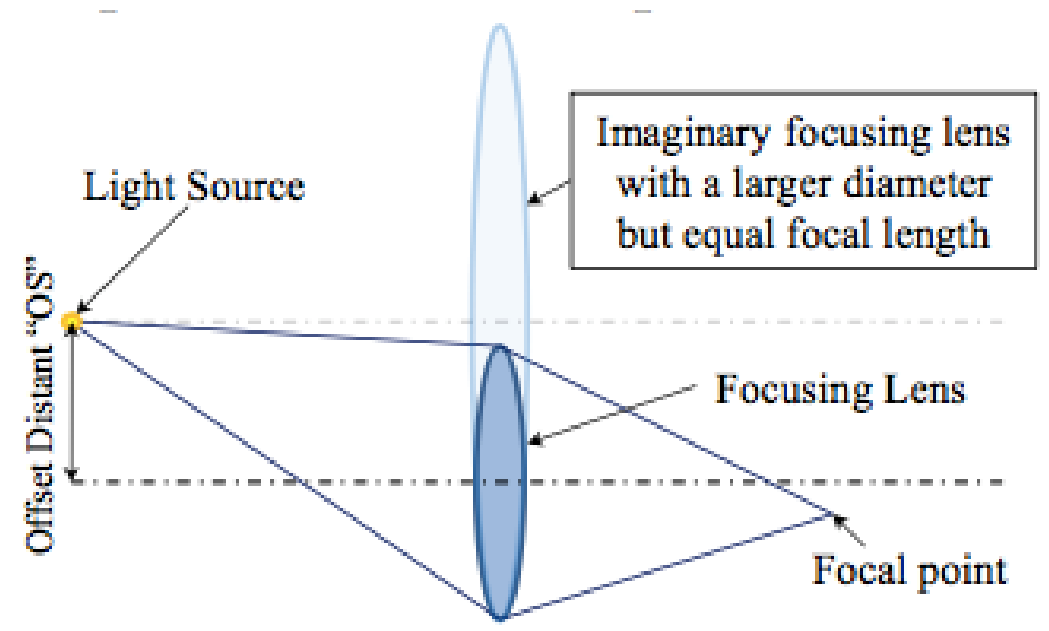

Figure 30: Light Source Offset Effect on the Effective F-number

(Wiley, 2017) $)^{35}$ 
The DOF of the current system is also investigated in this study. Optimization and evaluation of DOF are mentioned in the following chapter. 


\section{CHAPTER FOUR: OPTICAL DIAGNOSTICS}

As discussed in chapter one to two, it is beneficial to visualize the supersonic flow field for investigation of turbulent structure. In this chapter, optical flow field diagnostics and experimental procedures are discussed.

\section{Preliminary Testing}

To investigate the DOF of employed structured light-field focusing system, preliminary testing is conducted on benchtop before proceeding to wind tunnel testing. The investigation is carried out on a jet plume from an under-expanded jet. This jet is formed from a smooth brass tube with a $1.5 \mathrm{~mm}$ diameter and $5 \mathrm{~mm}$ of length. The air is exiting from the tube with a regulated supply pressure of 100 psi measured using an Omega ${ }^{\circledR}$ DPG1200-100 with 1.0\% full-scale accuracy. The jet is placed in the test section in Fig. 23 and visualized the density gradients in the plume. Since the control of two main parameters of the F-number $f^{\#}$ and the number of sources $N^{\#}$ is dominant to obtain thinner DOF, it is focused to modify these parameters in this testing. First, the array of LED bulbs is partially covered by electronic tapes to investigate the effect of light configurations on DOF, i.e., the effect of the number of sources. LEDs are mainly separated into three parts: outer diameter, inner diameter and center, as shown in Fig. 32. The red " $\mathrm{X}$ " marks in Fig. 32 indicate light sources not used. After the plane of best focus is determined, the jet is displaced until it appears blurred in the image. The focal length of the camera is initially fixed at $300 \mathrm{~mm}$. This testing is conducted visually to see if the image is defocused or still in focus and the DOF are 
obtained for each light configuration. To lessen the unwanted human error, this experiment is carried out three times for each testing. The exposure time is set at $7.15 \mathrm{~ms}$ and sampling rate of 139.9 Hz. This exposure time is changed for each light configuration respectively since it is not bright enough to illuminate the test section as the number of light sources decreases. For the inner light configuration, the exposure time of $7.15 \mathrm{~ms}$ is applied and $10.0 \mathrm{~ms}$ for the outer and the center.

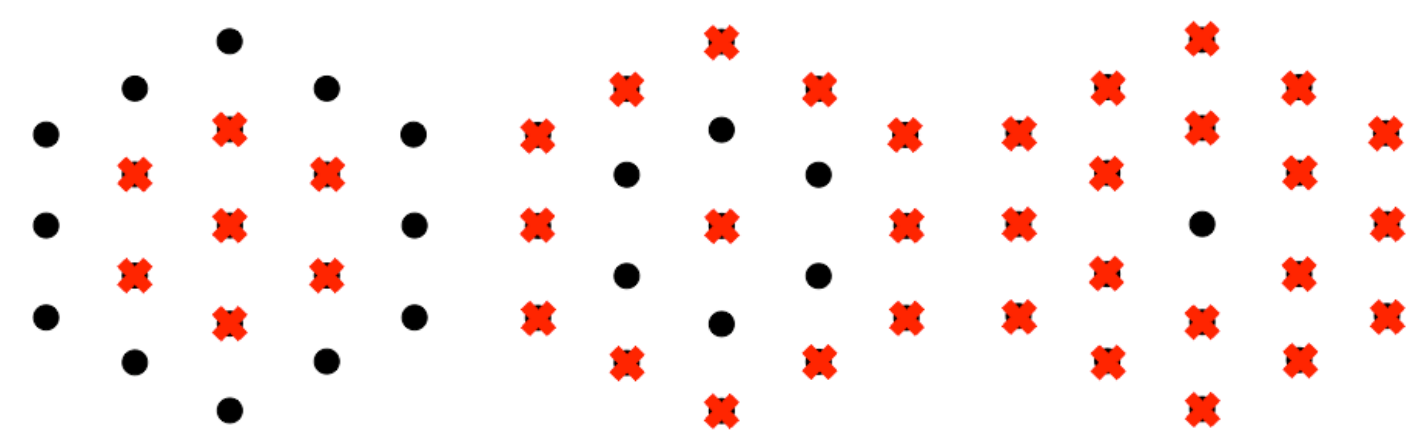

Outer Configuration Inner Configuration

Center Configuration

Figure 31: Light Configurations

Here, let us define $x$-direction in Fig. 23 along the optical axis and the plane of best focus is at $x=$ 0 . Both ends of DOF are, therefore, designated as positive and negative numbers of $x$ respectively. The measured DOF is summarized and shown in the following table. 
Table 2: DOF Measurements for Each Light Configuration

(Focal Length of $300 \mathrm{~mm}$ )

\begin{tabular}{|c|c|c|c|}
\hline & Positive $x(\mathrm{~mm})$ & Negative $x(\mathrm{~mm})$ & DOF $(\mathrm{mm})$ \\
\hline Outer & +0.423 & -0.677 & 1.10 \\
\hline Inner & +1.10 & -2.54 & 3.64 \\
\hline Center & +8.72 & -12.0 & 20.7 \\
\hline
\end{tabular}

As can be seen from this table, DOF is increased as the number of light sources decreases. This fact essentially indicates that the result from the previous work ${ }^{35}$ coincides with this one since the offset distance from the center axis OS in Fig. 31 increases as the number of sources increases. Taking the whole light configuration into consideration, the DOF of this current system is assumed as approximately less than $2 \mathrm{~mm}$. This fact also matches to Ahmed's work ${ }^{35}$.

Next, the focal length of the camera is decreased by increments of $50 \mathrm{~mm}$. It is important to note that the focusing lens is not replaced with different one, however, a regular digital camera has an imaging lens mounted inside and it is possible to imaginarily change the F-number of the system by modifying the focal length. The DOF with different F-numbers are, therefore, obtained in the same manner and summarized in following tables. Finally, to simplify the data to show these DOF numbers are put in a graph, as shown below. Since the DOF for the outer diameter light configuration is approximately $1.1 \mathrm{~mm}$ with $300 \mathrm{~mm}$ focal length, it can be assumed that the overall DOF with the whole LED bulbs is approximately $2 \mathrm{~mm}$. Similarly, it is determined to use the camera focal length of $300 \mathrm{~mm}$ due to the smallest DOF. As a reference, the images of jet taken during these experiments are also attached below. 
Table 3: DOF Measurements for Each Light Configuration

(Focal Length of $250 \mathrm{~mm}, 200 \mathrm{~mm}, 150 \mathrm{~mm}$ and $100 \mathrm{~mm}$ )

\begin{tabular}{|c|c|c|c|c|}
\hline $\begin{array}{l}\text { Focal Length } \\
\qquad(\mathrm{mm})\end{array}$ & $\begin{array}{c}\text { Light } \\
\text { Configuration }\end{array}$ & Positive $x(\mathrm{~mm})$ & Negative $x(\mathrm{~mm})$ & $\mathrm{DOF}(\mathrm{mm})$ \\
\hline \multirow{3}{*}{250} & Outer & +0.423 & -1.19 & 1.61 \\
\hline & Inner & +1.19 & -2.62 & 3.81 \\
\hline & Center & +11.2 & -13.6 & 24.8 \\
\hline \multirow{3}{*}{200} & Outer & +0.931 & -1.19 & 2.12 \\
\hline & Inner & +1.78 & -3.13 & 4.91 \\
\hline & Center & +12.3 & -14.2 & 26.5 \\
\hline \multirow{3}{*}{150} & Outer & +1.35 & -0.931 & 2.28 \\
\hline & Inner & +2.37 & -3.13 & 5.50 \\
\hline & Center & +18.3 & -17.0 & 35.3 \\
\hline \multirow{3}{*}{100} & Outer & +2.03 & -0.677 & 2.71 \\
\hline & Inner & +3.39 & -2.29 & 5.68 \\
\hline & Center & +19.5 & -17.9 & 37.4 \\
\hline
\end{tabular}




\section{Outer}

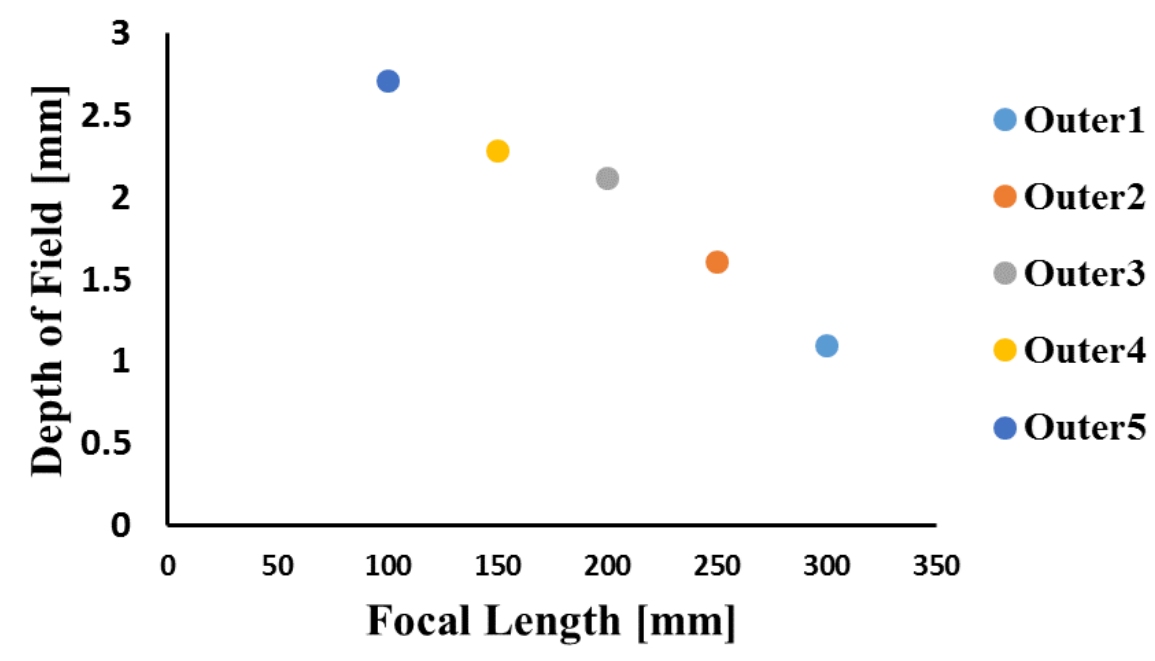

Figure 32: DOF for Outer Diameter Light Configuration

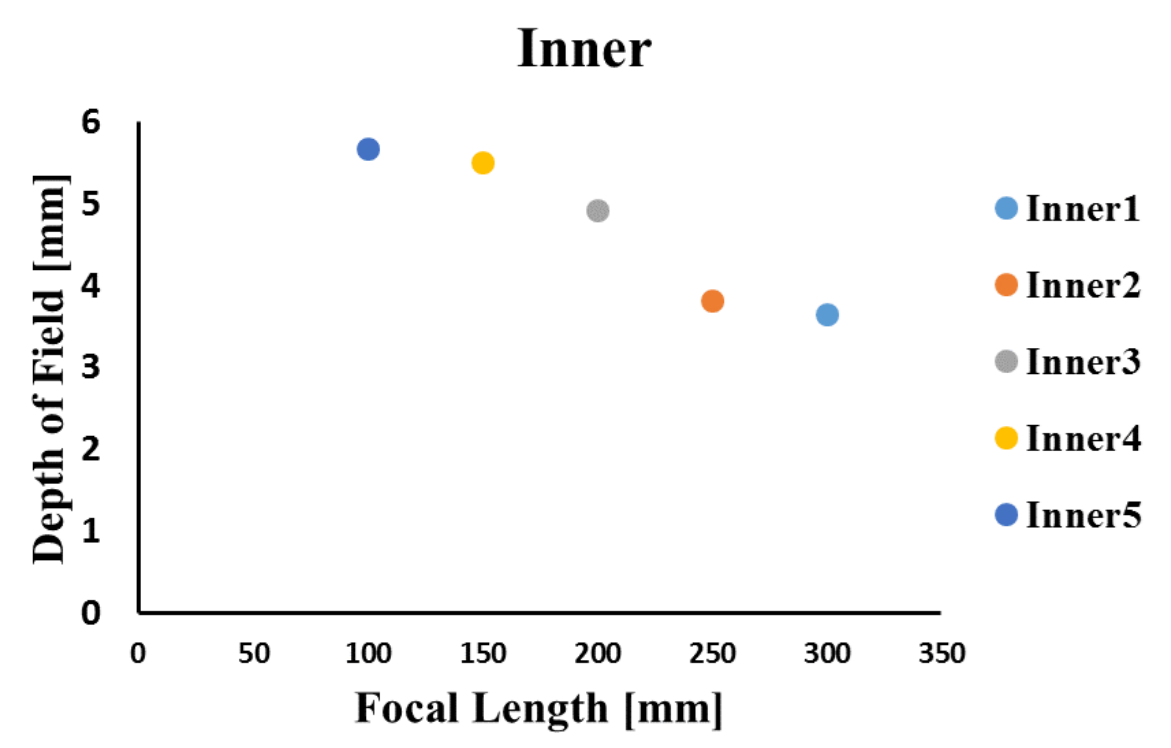

Figure 33: DOF for Inner Diameter Light Configuration 


\section{Center}

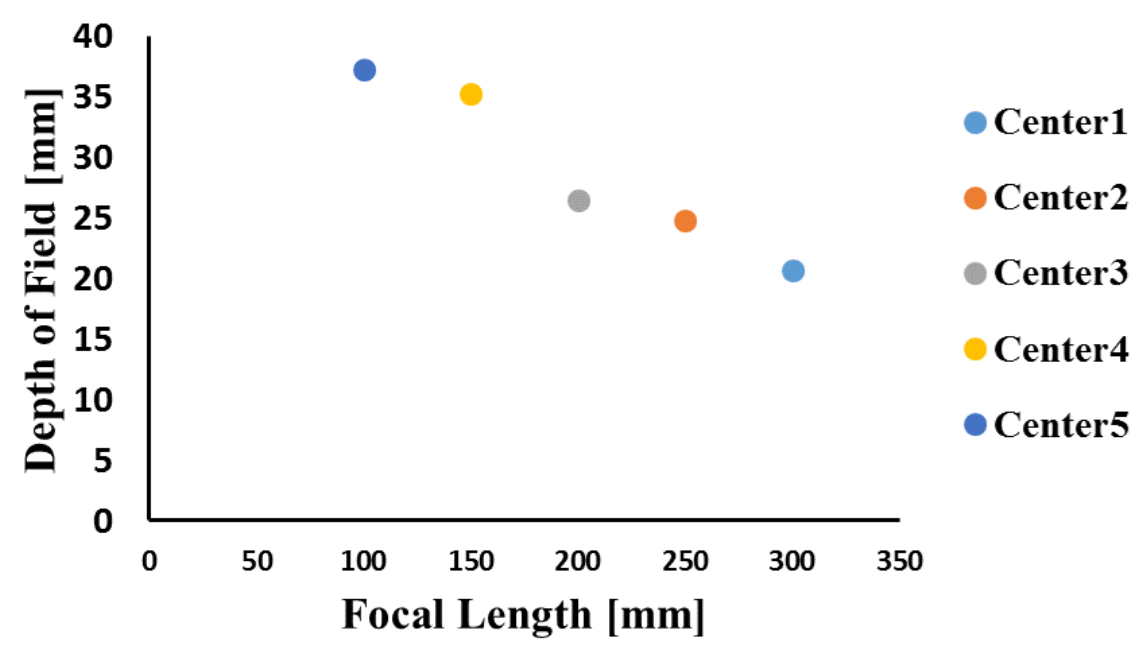

Figure 34: DOF for Center Light Configuration

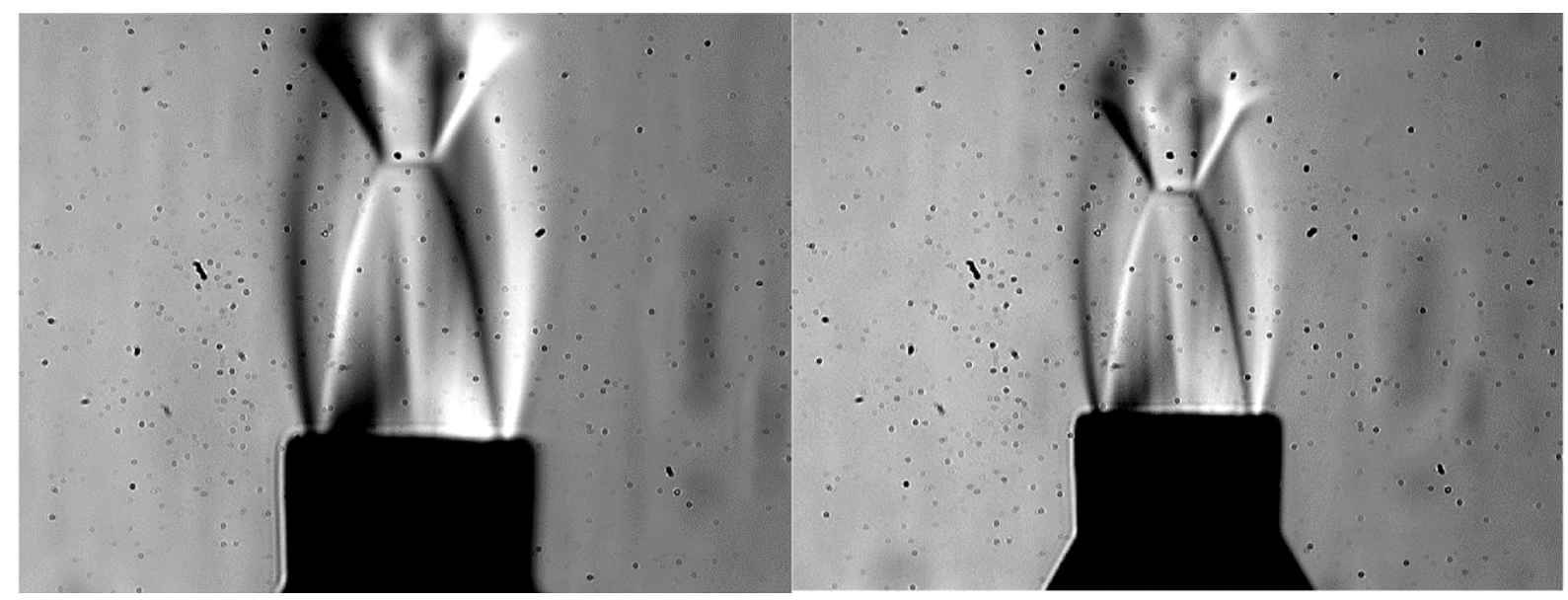

Figure 35: Under-Expanded Jet with 300mm(left) \& 250mm(right) Focal Length 


\section{$\underline{\text { Supersonic Wind Tunnel Testing }}$}

Since the investigation of DOF allows us to characterize the structured light-field focusing system, we now proceed to tests on the supersonic wind tunnel. The wind tunnel utilized in this work is a blow-down type facility with $100 \mathrm{~mm} \times 100 \mathrm{~mm}$ test window in the Center for Advanced Turbomachinery and Energy Research at the University of Central Florida. It is capable of achieving up to Mach 3.75 of flow and test duration of approximately 25 seconds. Also, it is possible to change Mach number by adjusting the throat area in this tunnel. A lever is attached to the throat section and it allows us of extrinsic access. In this study, it is targeted a flow of Mach 3.0 to visualize. A cone spike is mounted in the tunnel and the flow structure around the cone is observed using Structured Light-Field Focusing Schlieren. The following figure shows the schematic of the cone spike in the wind tunnel. It is custom designed from a stainless bolt of $7 \mathrm{~mm}$ diameter and is mounted at $50.6 \mathrm{~mm}$ height from the bottom of the tunnel with no angle of attack. The channel on the bolt head is filled with putty.

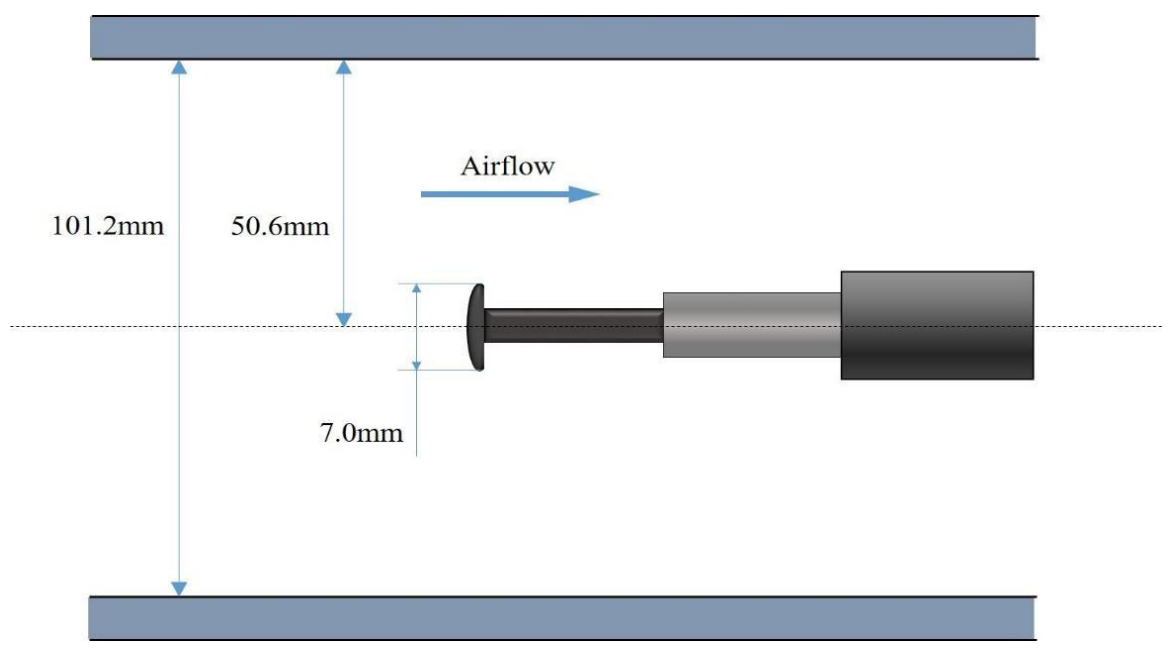

Figure 36: Cone Spike 
The air supplied from a compressor to the wind tunnel has stagnation pressure of $200 \mathrm{psi}$ in the chamber and it is sustained throughout the overall experiments. Note here that this compressor also supplies air to the entire laboratory. Thus, the stagnation pressure is dependent on how much air is being used and shared within the facility. For this reason, the total pressure is kept around 200 psi as close as possible. The throat area of the tunnel is fixed to achieve stationary Mach of 3.0. The camera has an exposure time of $6.3 \mathrm{~ms}$ and focal length of $70 \mathrm{~mm}$. DOF is calculated approximately $5 \mathrm{~mm}$ in this experiment. Here, the focal length is carefully chosen since when the camera is zoomed in too much, the tip of the cone can be a dominant region in the image. In that case, it can be in focus even after translating the whole setup, making it difficult to focus on a sliced shock. The procedure is that the setup is translated by $5 \mathrm{~mm}$ in one direction after capturing each sliced shock. This shock is observed through the camera soon after the chamber is opened to the atmosphere. Following images show the sliced shock at each location. The result is the cone is in focus and clearly visible initially. As translated by $5 \mathrm{~mm}$ in the image (b), it is slightly defocused. More translated at $10 \mathrm{~mm}$, this shock line becomes more blurry and transparent. This is because the initial shock line is getting out of focus. At $15 \mathrm{~mm}$, it splits into two shock lines and the one closer to the bolt head is focused more and the other is out of focus. This is considered that the camera captures the bow shock at a distant location while the initially focused shock appears in the background. This behavior can be seen more clearly in the image (e). At $25 \mathrm{~mm}$ translation, this focused shock becomes transparent since it is no longer in focus. White lines in images are due to dead pixels in the camera sensor. They are removed in the normalization process described below in next subchapter. 


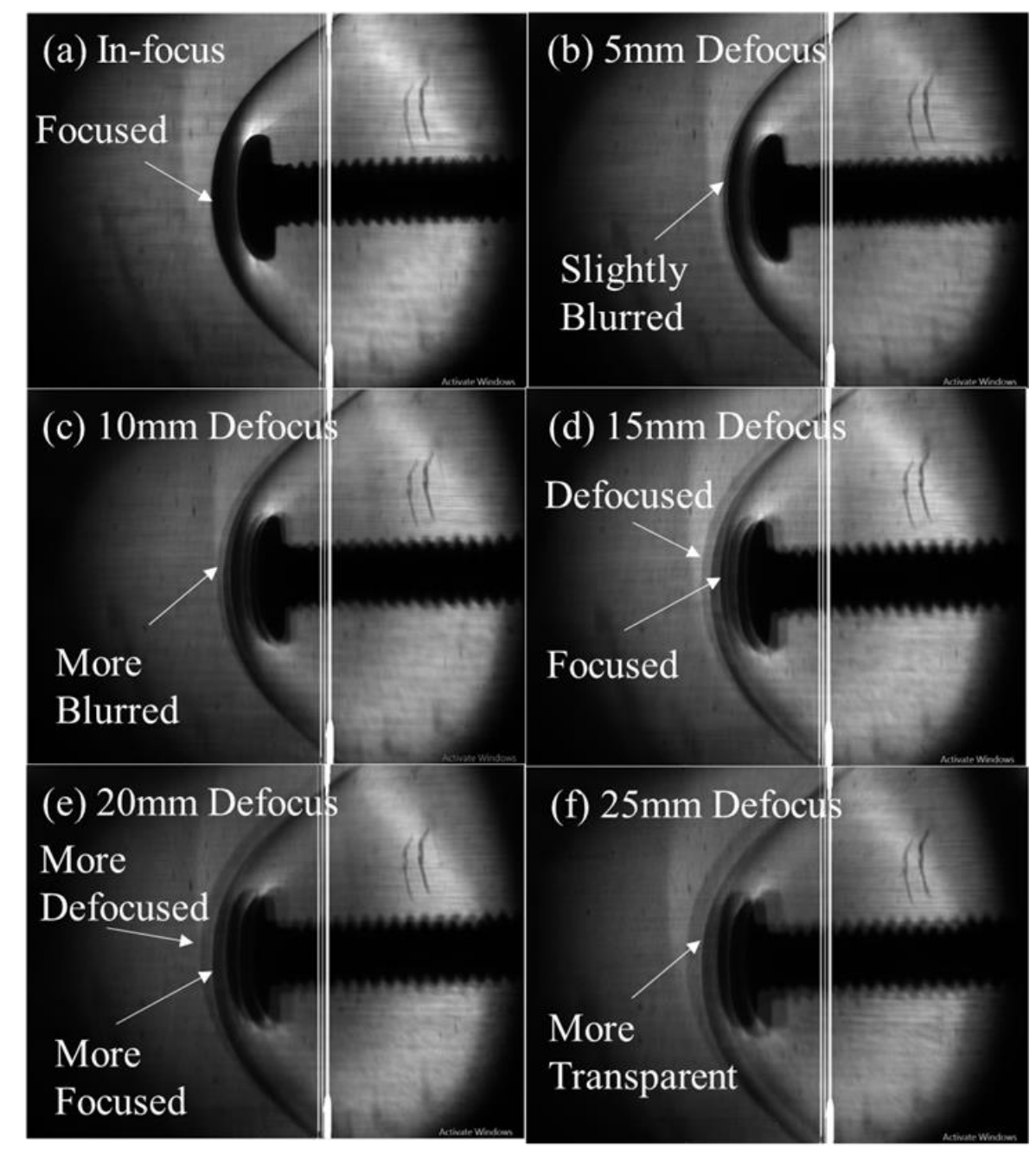

Figure 37: Sliced Shock lines at Each Translation

Also, the process of slicing shocks is briefly shown in the following diagrams. A 2D plane of focus which has $5 \mathrm{~mm}$ DOF sits at each translation position focusing on the partial shock structure. The intervals between these drawn planes are $5 \mathrm{~mm}$ in actual wind tunnel since the setup is translated. Although 2D planes are shown for both sides along the cone in this picture, the shock is measured on one side alone since it is estimated the structure is axis-symmetry. 


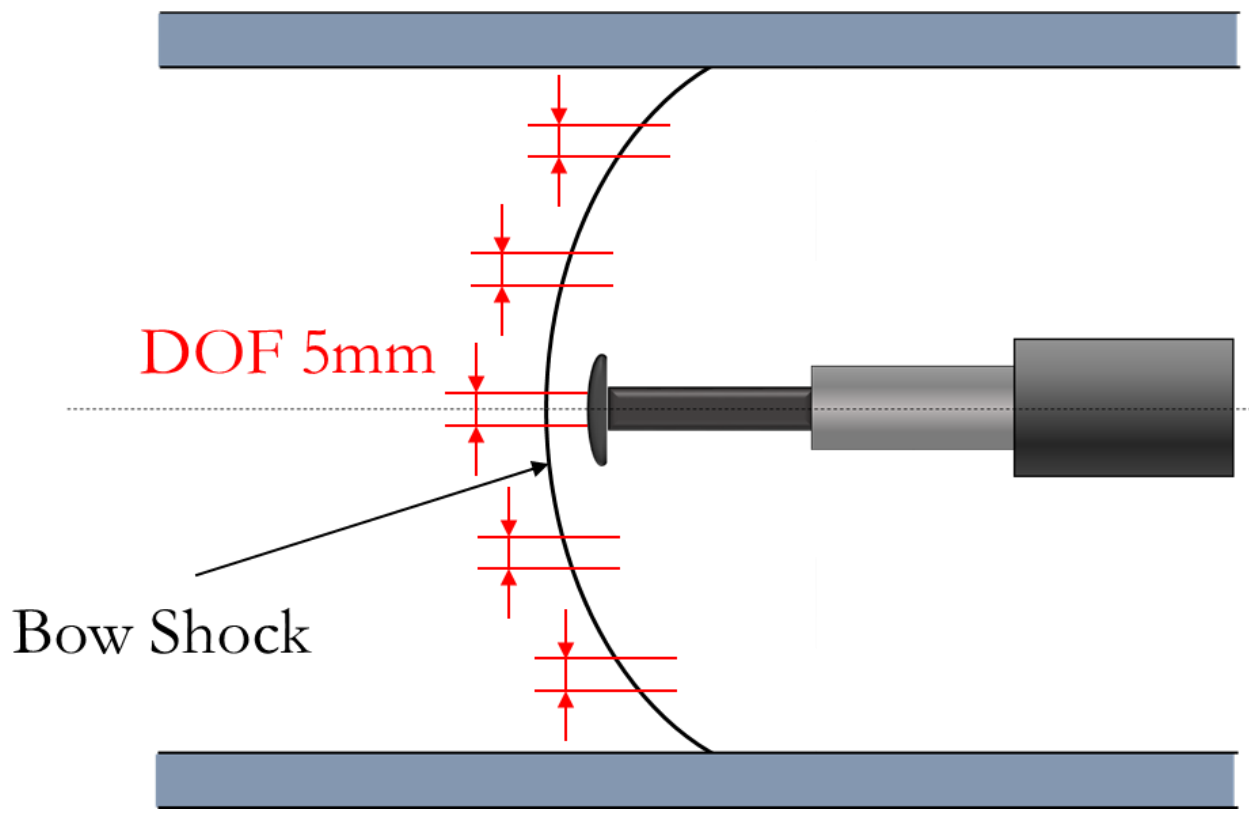

Figure 38: Process of Slicing Shocks 


\section{$\underline{\text { Post-Processing }}$}

After picturing the shock waves around the cone spike, those images are taken into MATLAB to process. Since the final goal of this study is solely the shocks, they are extracted by means of pixel division. First few paragraphs are spent on the explanation of this process in this chapter.

First, raw images of shock such as Figure 38 are loaded and kept in a file on MATLAB. In this software tool, grayscale images are recognized as matrices in which each cell has a certain value for light intensity. These values correspond to the brightness or contrast in the image and construct the actual loaded image. The raw images are uniformly cropped to the same size and mathematically added each other to calculate average pixel value. This is because the camera captures hundreds of shock images and choosing single image may cause a tremendous error in a final result. The crop size is 1200 by 936 as pixel resolution and the whole captured shock fits in this picture. This cropping process reduces unwanted background and computational cost. Also, the same steps are executed on the images with no flow. The cone with no shocks is photographed at each location where corresponds to the ones with air flow. First 5 of this bolt images are chosen to calculate average pixel value.

Second, these images without air flow are rotated and shifted in pixels to overlap the cone with images with shock. Cone shifting is caused due to strong air depletion onto the cone head resulting in a difference between images with and without shocks (Figure 40). In this picture, the cone and the shock are in focus and shown dark. A blue line represents the original cone location, 
thus it can be seen the cone is slightly shifted. This line is traced by means of edge tracking on MATLAB. The purpose of this process is to decrease this gap in cone location.

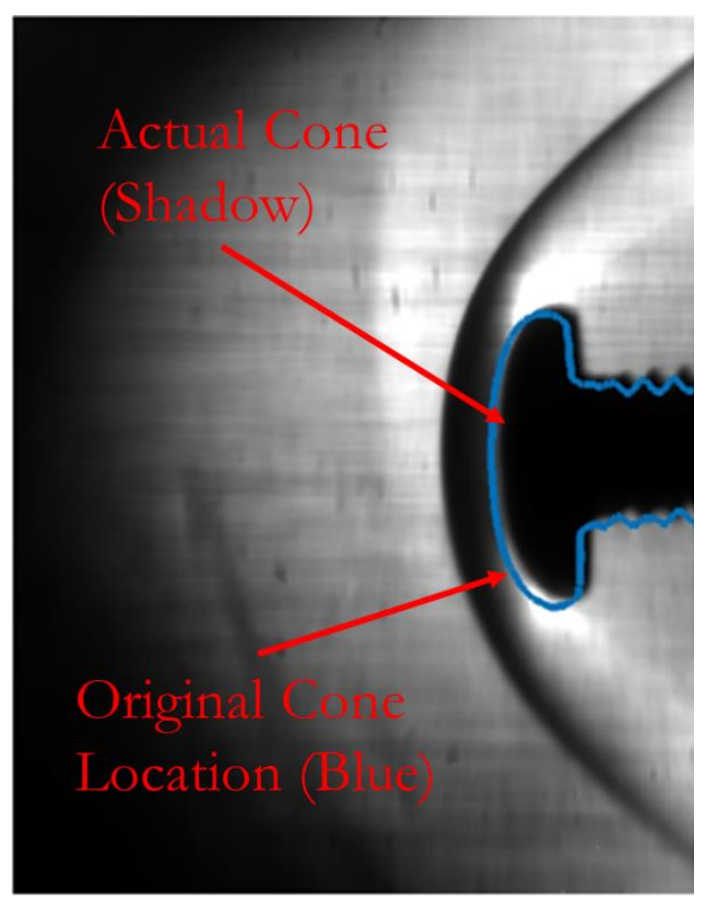

Figure 39: Cone Shifting in Pixel

It is relatively easy to see pixel changes in an image for the case of in-focus, however, it comes to difficult as the cone becomes defocused. Therefore, the amount of pixel shift and rotation which minimizes noise is respectively modified for each image.

Finally, the processed output images are mathematically divided by the processed reference images that have no shocks. As mentioned above, an image has certain values in pixel and can be mathematically processed with others when both images have the same pixel resolution. Each pixel value in the output image is respectively divided by the corresponding pixel in reference image 
resulting in an extracted shock. Since the difference between the output and the reference is solely shock, pixel values other than this region should be nearly one. Therefore, the shock is exclusively shown as white while it is black in the background. The contrast in the result images is uniformly adjusted not to be saturated. Each processed image is placed according to the actual position in the wind tunnel in the following figure. The shock in front of the cone head appears in focus initially and becomes defocused at a distant location as the same as seen in Figure 38.

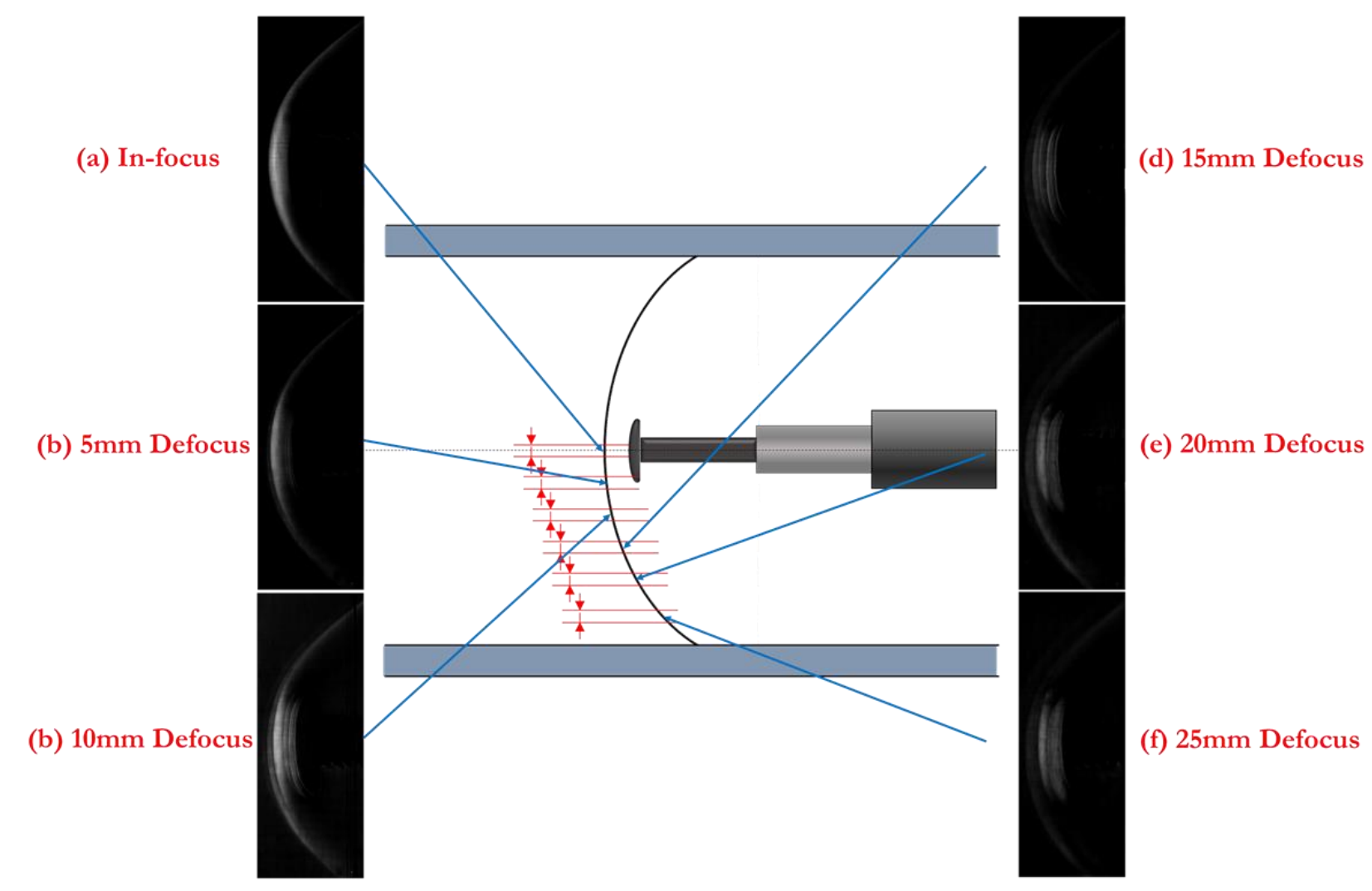

Figure 40: Shocks at Different Location 
Now, 2D shock images are ready to reconstruct 3D shock structure. To simplify the reconstruction process, a leading edge is traced from each processed shock image. This is done by the same approach as edge tracking. Similar to Figure 40, a blue line represents the shock in the following image.

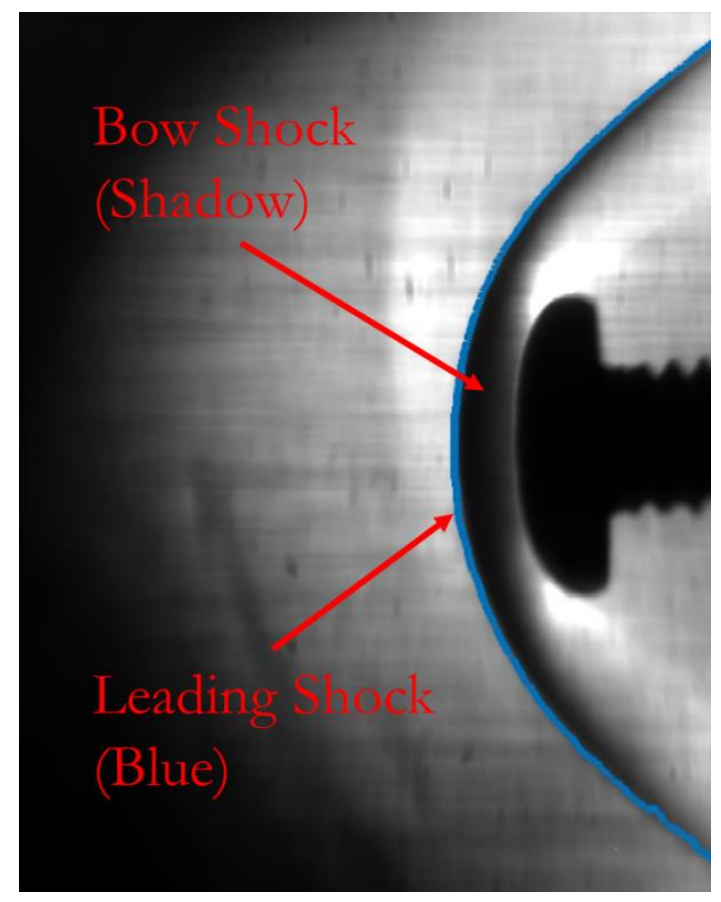

Figure 41: Edge of Leading Shock

Figure 42 shows the leading shock in focus. Similarly, each edge is traced for other shocks observed at different locations. Curve fitting is then applied to these lines to find a mathematical function that has the best fit to this series of the data point. These fitted edges are used in the 3D reconstruction of shock structure described in the following chapter. 


\section{CHAPTER FIVE: 3D RECONSTRUCTION}

In this chapter, a 3D model of shock structure is reconstructed. It is based on some assumptions made in this experiment. Since the cone can be considered symmetry, it can be assumed that the shock induced around it is also perfect symmetry. Therefore, an estimate of 3D shock structure is created by revolving the leading shock in focus around its axis. The shock in focus is expressed in a mathematical function and revolved in a polar coordinate. It is rotated by 0 to 180 degrees in an interval of 500. Thus, there should be 500 lines in $3 \mathrm{D}$ coordinate representing each rotated shock lines by the above resolution. A surface is then created using a surf function in MATLAB that complements what is missing between each line. This surface is the estimate of shock structure and shown gray with certain transparency for some convenience.

The second assumption is that each leading shock taken at different position fits the surface of this revolved 3D plot. This means the actual shock edges should mathematically correspond to the intersections where the estimate is sliced at the respective location. In the following figure, the estimate of shock structure and each leading edge are plotted. The gray object represents the estimate and red lines are leading shocks at each position. Note here that the shock at $10 \mathrm{~mm}$ or further are not shown in this figure since they are too far to render and it may increase computational cost. The red lines are placed according to the spatial resolution described in the previous chapter. The size of the cone head is $7 \mathrm{~mm}$ as mentioned and the pixel resolution of this head is approximately 400 , then the spatial resolution is calculated as 0.0175 . 


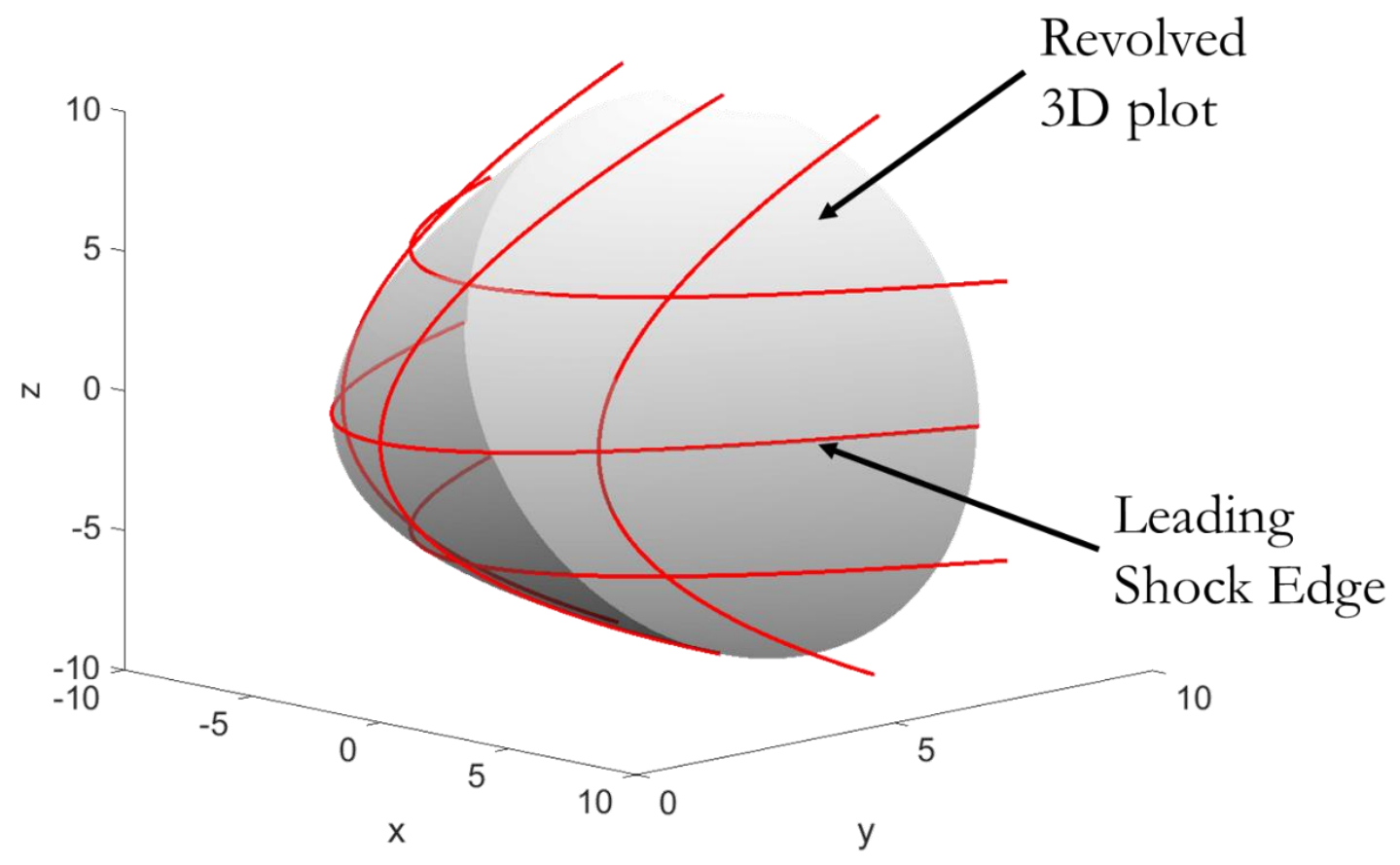

Figure 42: 3D Reconstruction of Shock Structure (XYZ view)
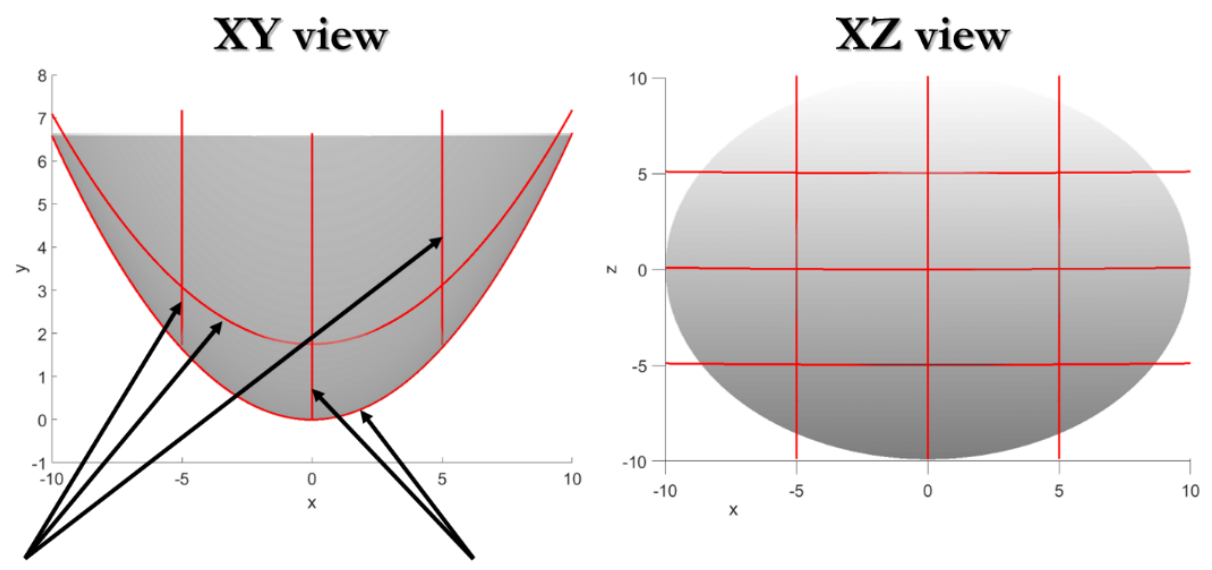

Sliced Shock

Sliced Shock at $\pm 5 \mathrm{~mm}$ in Focus

Figure 43: 3D Reconstruction of Shock Structure (XY view \& XZ view) 
Three line at $x=+5$ is initially plotted and then flipped to the other side which technically corresponds to $x=-5$ since the model is estimated symmetry. All three lines are rotated 90 degrees to show similar trends in this picture.

Although it is assumed that the shock at $\pm 5 \mathrm{~mm}$ fits the surface of the estimate, it is slightly off as can be seen in Figure 43. This is considered as an error between the actual data and the estimate. A plane at $z=+5$ which coincides with the estimate is created to plot intersecting surface. This surface is outlined by a specific mathematical function plotted in Figure 46 with the actual data at $\pm 5 \mathrm{~mm}$ defocus. Each $x$ position of both plots is compared and calculated how far this actual plot is detached from the estimate. Assuming these plots are symmetry, the error is calculated as approximately $9.64 \%$.

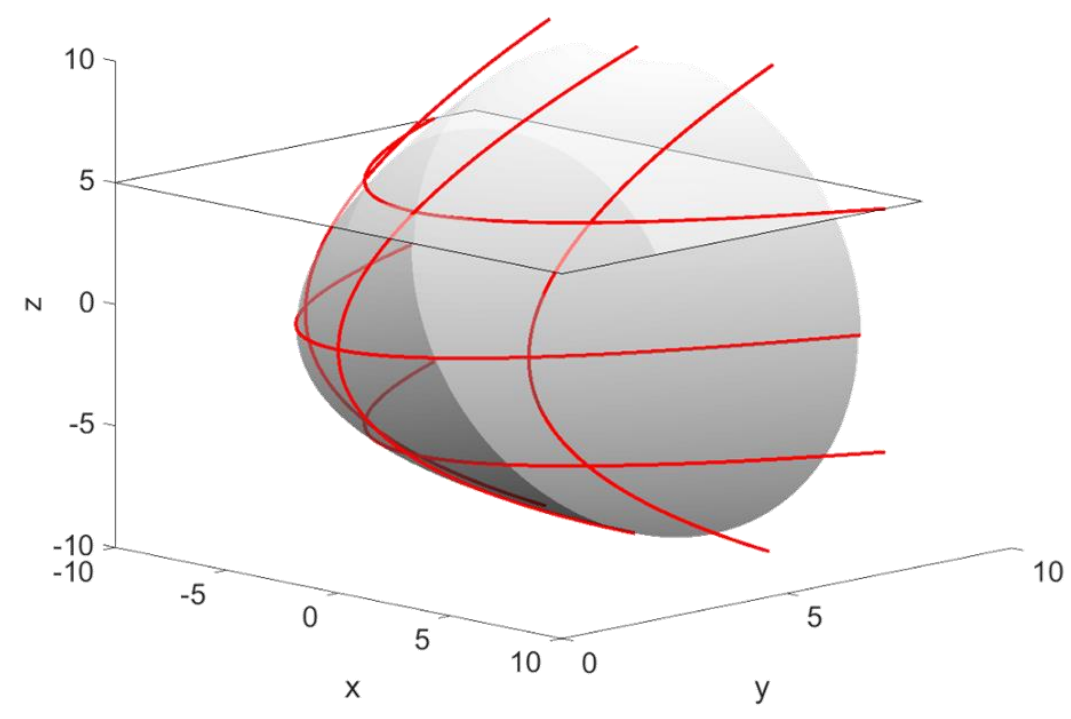

Figure 44: 3D model with an Intersecting Plane 


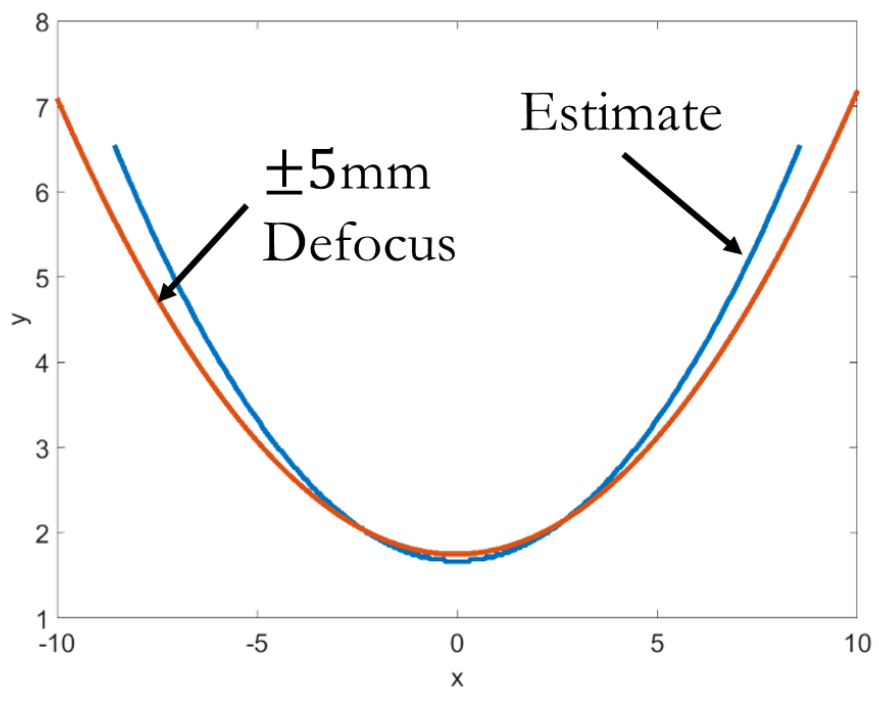

Figure 45: Uncertainties

It can be considered that the error is caused due to large oscillations while wind tunnel operation which might result in a slight offset in the optical setup. Also, the shock structure is assumed complete symmetry which does not necessarily coincide with the one in reality. Constructing more robust optical system can improve this error against the oscillations. 


\section{CHAPTER SIX: CONCLUSIONS}

This research is focused on reconstructing a 3D model of shock structure using the Structured Light-Field Focusing Schlieren (SLFF). The diagnostic technology is formulated based on light field principle and multiple light sources to generate the 2D planar focusing effect. Unlike conventional focusing schlieren system, SLFF utilizes discrete light sources that greatly simplify the optical setup and alignment process. As described throughout this report, SLFF system can be optimized using some control parameters such as a number of light sources and different lens fnumbers. They allow us to modify system depth of focus (DOF) and apply SLFF to specific research requirements. Lastly, 3D shock structure induced around a cone spike is successfully reconstructed and it proves the feasibility of SLFF as a flow diagnostic tool. 


\section{LIST OF REFERENCES}

${ }^{1}$ Hatill, W. B., “Analytical and Experimental Investigation of a Scramjet Inlet of Quadriform Shape," U. S. Air Force, TR AFAPL-TR-65-74, Marquardt Corp., August, 1965.

${ }^{2}$ Kutshenreuter, P. H., "Hypersonic Inlet Tests in Helium and Air," AIAA Propulsion Joint Specialist Conf., U. S. Air Force Academy, CO, June, 1965.

${ }^{3}$ Kiersey, J. L. and Snow, M. L., "Modular Inlet Investigation," Aeronautics Div., Research and Development, Applied Physics Lab., Quarterly Rept. AQR/66-1, Johns Hopkins Univ., Baltimore, MD, January, 1966.

${ }^{4}$ Holland, S. D., "Internal Aerodynamics of a Generic Three-Dimensional Scramjet Inlet at Mach 10," NASA Technical Paper, 3476, February. 1995.

${ }^{5}$ Smart, M. K., "Design of Three-Dimensional Hypersonic Inlets with Rectangular-to-Elliptical Shape Transition," Journal of Propulsion and Power, vol. 15, No. 3, June. 1999.

${ }^{6}$ Gladstone, J. H. and Dale, T. P., "Researches on the Refraction, Dispersion and Sensitiveness of Liquids," Phil. Trans. Royal Soc, 153, 317-343, London, 1864.

${ }^{7}$ Hooke, R., "Of a New Property in the Air," Micrographia, Observation LVIII, 217-219, London, 1665.

${ }^{8}$ Toepler, A., "Beobachtungen nach einer neuen optischen Methode," Maximillan Cohen und Sohn, Bonn, 1864.

${ }^{9}$ Settles, G. S., "Schlieren and Shadowgraph Techniques," Springer, Berlin, pp. 1-24, Heidelberg and New York, 2001.

${ }^{10}$ Speak, G. S. and Walters, D. J., "Optical Considerations and Limitations of the Schlieren Method,” British Aeronautical Research Council, Report No. 2859, 1954. 
${ }^{11}$ Kantrowitz, A. and Trimpi, R. L., “A Sharp-Focusing Schlieren System,” Journal of the Aeronautical Sciences, vol. 17, pp. 311-314, 319, 1950.

${ }^{12}$ Weinstein, L. M., “An Improved Large-Field Focusing Schlieren System,” AIAA Paper 91 0567, January, 1991.

${ }^{13}$ Gartenberg, E., Weinstein, L. M. and Lee Jr., E. E., “Aerodynamic Investigation with Focusing Schlieren in a Cryogenic Wind Tunnel," AIAA Journal, vol. 32, No. 6, pp. 1242-1249, 1994.

${ }^{14}$ Alvi, F. S., Settles, G. S. and Weinstein, L. M., “A Sharp-Focusing Schlieren Optical Deflectometer," AIAA paper 93-0629, 1993.

${ }^{15}$ Kashitani, M. and Yamaguchi, Y., "Flow Visualization around a Double Wedge Airfoil Model with Focusing Schlieren System,” Journal of Thermal Science, vol. 15, No. 1, 2006.

${ }^{16}$ Cook, W. J., Presley, L. L. and Chapman, G. T., "Shock Tube as a Device for Testing Transonic Airfoils at High Reynolds Numbers,” AIAA Journal, 17(7): 714-721, 1979.

${ }^{17}$ Kashitani, M., Yamaguchi, Y. and Nakao, S., "Visualization of a Double Wedge Airfoil Model in a Transonic Shock Tube Flow,” NCTAM2003, 307-308, 2003.

${ }^{18}$ Kashitani, M., Yamaguchi, Y. and Saito, T., "Observation of Shock Tube Airfoil Flow with a Sharp Focusing Schlieren Method," Theoretical and Applied Mechanics, 49: 249-255, 2000.

${ }^{19}$ Toshinori, K., Christopher, P. G. and Robert, D. R., "Focusing-schlieren Visualization in a Dual-mode Scramjet," Exp Fluids, 56:211, 2015.

${ }^{20}$ Goulding, J. S., “A Study of Large-scale Focusing Schlieren System,” University of the Witwatersrand, Johannesburg, 2006. 
${ }^{21}$ Faraday, M., “Thoughts on Ray Vibrations,” Philosophical Magazine, S. 3, Vol. 28, N188, 1846.

${ }^{22}$ Gershun, A., "The Light Field," Journal of Mathematics and Physics, Vol. 18, MIT, pp. 51151, 1939.

${ }^{23}$ Levoy, M., “Light Fields and Computational Imaging,” IEEE Computer Society, August, 2006.

${ }^{24}$ Gortler, S. J., Grzeszczuk, R., Szeliski, R. and Cohen, M. F., “The Lumigraph,” Proc. ACM Siggraph, ACM Press, pp. 43-54, 1996.

${ }^{25}$ Benton, S. A., "Survey of Holographic Stereograms," Proceedings of the SPIE, 391, 15-22, 1982.

${ }^{26}$ Arvo, J., "The Irradiance Jacobian for Partially Occluded Polyhedral Souces," Proc. ACM Siggraph, ACM Press, pp. 335-342, 1994.

${ }^{27}$ Levoy, M. and Hanrahan, P., “Light Field Rendering,” Proc. ACM Siggraph, ACM Press, pp. 31-42, 1996.

${ }^{28}$ Adelson, E. H. and Wang, J. Y. A., "Single Lens Stereo with a Plenoptic Camera," IEEE Transactions on Pattern Analysis and Machine Intelligence 14, 2, 99-106, February, 1992.

${ }^{29} \mathrm{Ng}$, R., Levoy, M., Brédif, M., Duval, G., Horowitz, M. and Hanrahan, P., "Light Field Photography with a hand-held Plenoptic Camera," Stanford University Computer Science Tech Report CTSR 2005-02, April, 2005.

${ }^{30}$ Thurow, B. S. and Fahringer, T. W., "Recent Development of Volumetric PIV with a Plenoptic Camera," 10th International Symposium On Particle Image Velocimetry - PIV13, Delft, the Netherlands, July, 2013. 
${ }^{31}$ Fahringer, T. W., Lynch, K. P. and Thurow, B. S., "Volumetric Particle Image Velocimetry with a Single Plenoptic Camera," Measurement Science and Technology, 26, 115201, pp. $25,2015$.

${ }^{32}$ Johnson, K. C., Thurow, B. S., Kim, T., Blois, G. and Christensen, K. T., "Three Dimensional Plenoptic PIV Measurements of a Turbulent Boundary Layer Overlying Rough and Permeable Surfaces," 18th International Symposium on the Application of Laser and Imaging Techniques to Fluid Mechanics, Lisbon, Portugal, July, 2016.

${ }^{33}$ Fahringer, T. W., Thurow, B. S., Humphreys, W. M, Jr. and Bartram, S. M., "Comparison of Stereo-PIV and Plenoptic-PIV Measurements on the Wake of a Cylinder in NASA Ground Test Facilities,” AIAA SciTech, NF1676L-24762, January, 2017.

${ }^{34}$ Fahringer, T. W. and Thurow, B. S., "Filtered Refocusing: a Volumetric Reconstruction Algorithm for Plenoptic-PIV," Measurement Science and Technology, 27, 094005, pp. 14, 2016.

${ }^{35}$ Ahmed, K. and Wiley, A., "Structured Light-Field Focusing for Flowfield Diagnostics," Experimental Thermal and Fluid Science, 1-14, 2017. 